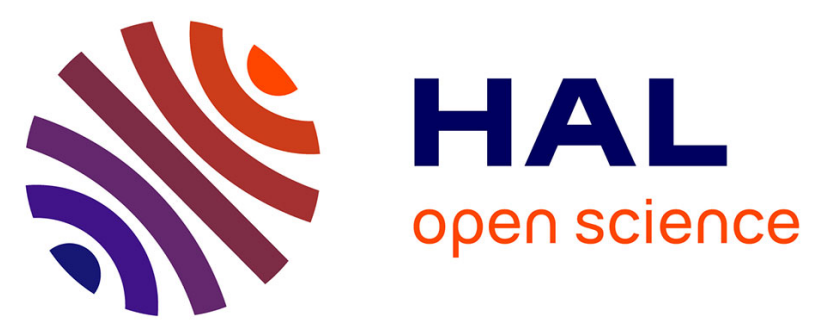

\title{
Chitosan based-nanoparticles and nanocapsules: Overview, physicochemical features, applications of a nanofibrous scaffold, and bioprinting
}

Kamel R Shoueir, Nagwa El-Desouky, Moataz M Rashad, M.K. Ahmed, Izabela Janowska, Maged El-Kemary

\section{To cite this version:}

Kamel R Shoueir, Nagwa El-Desouky, Moataz M Rashad, M.K. Ahmed, Izabela Janowska, et al.. Chitosan based-nanoparticles and nanocapsules: Overview, physicochemical features, applications of a nanofibrous scaffold, and bioprinting. International Journal of Biological Macromolecules, 2021, 167, pp.1176-1197. 10.1016/j.ijbiomac.2020.11.072 . hal-03429245

\section{HAL Id: hal-03429245 \\ https://hal.science/hal-03429245}

Submitted on 15 Nov 2021

HAL is a multi-disciplinary open access archive for the deposit and dissemination of scientific research documents, whether they are published or not. The documents may come from teaching and research institutions in France or abroad, or from public or private research centers.
L'archive ouverte pluridisciplinaire HAL, est destinée au dépôt et à la diffusion de documents scientifiques de niveau recherche, publiés ou non, émanant des établissements d'enseignement et de recherche français ou étrangers, des laboratoires publics ou privés. 
1 Chitosan based-nanoparticles and nanocapsules: Overview, physicochemical

2 features, applications of a nanofibrous scaffold, and bioprinting

3 Kamel R. Shoueir ${ }^{a, b}$, Nagwa El-Desouky, Moataz M. Rashad ${ }^{a}$, M. K. Ahmed ${ }^{c}$, Izabela

4 Janowskab ${ }^{b}$ Maged El-Kemary ${ }^{a, d}$

$5{ }^{a}$ Institute of Nanoscience \& Nanotechnology, Kafrelsheikh University, 33516 Kafrelsheikh,

$6 \quad$ Egypt

7 b Institut de Chimie et Procédés pour l'Énergie, l'Environnement et la Santé (ICPEES), CNRS

8 UMR 7515-Université de Strasbourg, 25 rue Becquerel 67087 Strasbourg, France

$9 \quad{ }^{c}$ Department of Physics, Faculty of Science, Suez University, Suez, 43518, Egypt

$10{ }^{\mathrm{d}}$ Pharos University, Alexandria, Egypt

11 Corresponding author:

12 *Kamel Shoueir(Ph.D.); kamel_rezk@nano.kfs.edu.eg

$13 * *$ Maged El-Kemary (Ph.D.); elkemary@nano.kfs.edu.eg

14 Abstract

15 Recent advancements in the synthesis, properties, and applications of chitosan as the second after 16 cellulose available biopolymer in nature were discussed in this review. A general overview of 17 processing and production procedures from $\mathrm{A}$ to $\mathrm{Z}$ was highlighted. Chitosan exists in three polymorphic forms which differ in degree of crystallinity $(\alpha, \beta$, and $\gamma)$. Thus, the degree of deacetylation, crystallinity, surface area, and molecular mass significantly affect most applications. Otherwise, the synthesis of chitosan nanofibers is suffering from many drawbacks that were recently treated by co-electrospun with other polymers such as polyvinyl alcohol (PVA), polyethylene oxide (PEO), and polycaprolactone (PCL). Ultimately, this review focuses on the area of new trend utilization of chitosan nanoparticles as nanospheres and nanocapsules, in cartilage and bone regenerative medicine. Owing to its biocompatibility, bioavailability,

25 biodegradability, and costless synthesis, chitosan is a promising biopolymeric structure for water remediation, drug delivery, antimicrobials, and tissue engineering. 
27 Keywords: Chitosan; chitosan production; the degree of deacetylation; chitosan physicochemical properties; chitosan nanofibers; bioprinting.

\section{Chitosan source}

Chitosan consists of residues of 2-acetamido-2-deoxy- $\beta$-D-glucopyranose and 2-amino-2deoxy- $\beta$-D-glucopyranose (Fig. 1) and it is the second prevalent biopolymer in nature after cellulose. The $-\mathrm{NH}_{2}$ and $-\mathrm{OH}$ in the chitosan structure are the main functional groups responsible for chitosan properties and its unlimited application. In 1859, Rouget discovered chitosan when he studied the deacetylated forms of chitin from crustacean marine [1]. Since the first international conference of chitin and chitosan (Boston, USA, 1977), scientific and industrial communities have shown exponential interest in chitin and chitosan as a costless biopolymeric structural form [2].

Crustaceans, insects, mollusks, and fungi are the main sources of chitosan. In industry, chitosan is made from the external chitinous exoskeleton of marine crustaceans such as crabs, shrimps, lobsters, crayfish, krill, and woodlice which are waste products that originated from the fishing industry [3]. First, the crude material is subjected to demineralization, deproteinization, deodorization, and drying to extract chitin. Then, the chitin is being deacetylated, cleaned, and left to dry to gather chitosan. This method is economically efficient since raw materials are not associated with any costs and could include the extraction of Astaxanthin. Currently, chitosan is commercially produced in the entire world and its price in powder form depends on the application, properties, and purity. The main characteristics that ascertain the biopolymer of chitosan are its molecular weight and the degree of deacetylation. Other properties are important, such as crystallinity, surface area, particle size, air humidity, and ash content [4, 5]. These characteristics are important in controlling the properties of chitosan, i.e. solubility, polycation nature, antimicrobial activity, biocompatibility, and bioadhesion. Consequently, different shapes

51 and physical forms of chitosan such as films, nanofibers, nanoparticles, nanocapsules,

52 microparticles, membranes, sponges, scaffolds, and hydrogels determine their possible utilization 53 in water treatment, food processing, cosmetics, agriculture, and biomedicine [6-8].

\subsection{Processing and production of chitosan}


In general, two main processes are used for chitosan production: the ordinary process and the fermentation process [9]. In the ordinary method, the sources are crustaceans, insects, or mollusks. First, chitin is isolated from the raw materials using various chemical treatments that remove ash, proteins, color, and flavor. Chitin is converted to chitosan by alkaline deacetylation. Several phases are performed to purify chitosan such as dissolution, precipitation, centrifugation, and finally, the drying phase. In the fermentation process, chitosan is obtained from fungi, i.e. fungal biomass containing glucan complexes with chitin or chitosan is obtained by fermentation, [10]. Then the biomass is separated from the fermentation medium by filtration and drying and the next successive steps are performed to extract the chitosan [11]. Fig. 2 shows the main steps of the ordinary method to produce chitosan. This process usually begins with the demineralization phase. The main goal of this step is to reduce the ash and mineral content in the crustacean waste. Ash mainly consists of inorganic traces such as carbonates, phosphates, and other mineral salts [12]. Demineralization occurs mainly in dilute $\mathrm{HCl}$ solutions (1-10\%) assisted by stirring at room temperature for short periods of nearly 1-3 hours [13]. This treatment guarantees complete removal of the mineral content without destroying the polymer chains of the chitin. Other acids like nitric acid, sulfuric acid, formic, acetic, and EDTA can be also used [14]. Unfortunately, these nominated acids are effective, but they can damage the structure of chitin in the depolymerization process. The second step is deproteinization that includes the removal of proteins residues adhered to the demineralized powder. It proceeds with alkalis including sodium hydroxide, sodium bicarbonate, sodium carbonate, potassium carbonate, potassium hydroxide, or calcium hydroxide. The most common option is the use of sodium hydroxide (1-10\%) with a gentle stirring at a temperature that reaches $100{ }^{\circ} \mathrm{C}$ and the period between 2 and 72 hours. A deproteinization requires effective control of time and temperature, as high temperatures and longer periods can lead to depolymerization and deacetylation. On the other hand, enzymatic treatment with proteases enzymes such as pepsin and trypsin is an alternative form of deproteinization but less efficient than alkaline treatment [15].

The third step in the isolation of chitin is deodorization/depigmentation responsible for removing pigments and odors. For this purpose, ethanol, acetone, potassium permanganate, sodium hypochlorite, or hydrogen peroxide are generally used [16]. It is important to notify that the above-mentioned phases are followed by several successive washing processes up to obtaining a controlled neutral $\mathrm{pH}$ value. The final product of demineralization, deproteinization, 
and deodorization/depigmentation is chitin. It can then be dried for later use or immediately

87 deacetylated.

88 The deacetylation reaction is the most important step in this processing, at this stage, chitin turns into chitosan. During the deacetylation of the chitin C-2 position, the acetamide group is converted into amino groups $\left(-\mathrm{NH}_{2}\right)$ [17]. When the percentage of amino groups attains around $50 \%$ or more, the polymer becomes soluble in aqueous acidic media, and in this case, chitosan can be obtained [18]. Alkaline hydrolysis with sodium hydroxide (40-50\%) at high temperatures (80-120 ${ }^{\circ} \mathrm{C}$ ) is the most common method for extracting chitosan from chitin [19]. The main factors influencing the deacetylation reaction are the alkali concentration, reaction time, solid/liquid ratio, temperature, and the size of the chitin particles. Several alternative methods have been studied to improve or replace conventional alkaline hydrolysis, including enzymatic agents, vapor explosion, microwave deacetylation, and ultrasonic deacetylation [20].

The purification step can be performed to produce high purity chitosan. In this case, the deacetylation product is dissolved in an acidic medium, obtaining a viscous solution with an insoluble fraction consisting of impurities, mainly ash, which has not been completely removed in the demineralization step. This viscous solution is centrifuged or filtered, and the insoluble fraction is discarded. The alkali is then added to the viscous solution, and consequently, a suspension with precipitated chitosan is formed. The suspension is centrifuged again to obtain pure chitosan (90-95\%) in the form of a paste. The previous steps (dissolution / centrifugation / precipitation / centrifugation) can be repeated several times to obtain chitosan with high purity (purity greater than 99.9\%) [21].

Drying is the last step and is necessary to obtain pure chitosan. This process is important to produce chitosan and to provide the moisture content which is required to conserve the product without changing the material. Typically, after drying, the desired product should have a moisture content of less than $10 \%$ (in the wet state) to provide good physicochemical and microbiological aspects after long-term storage. Polymerization reactions and Maillard reactions are the most important changes that should be avoided during the drying operation. Some methods, such as convection, floor drying, drying in the nozzle layer, spray drying, sun drying, oven drying, infrared drying, freeze-drying, and low-pressure overheating have been used to dry chitosan. All 
115 these methods have their advantages and disadvantages, while air, temperature, and residence

116 time are the key factors that must be controlled to obtain a good quality of chitosan product [22].

\section{1.2. Chitosan characteristics}

The main characteristics that determine the quality and flow rate of chitosan are the molecular weight, degree of deacetylation, crystallinity, surface area, and size of the particles as mentioned before [23]. Herein, the molecular mass of chitosan is a characteristic associated with the number of monomer units of a biopolymer. The evaluation and modification of this property are very important since molecular weight influences the properties like viscosity and solubility [24]. In general, the chitosan molecular weight ranges from 20 to $1200 \mathrm{kDa}$. Based on the molecular weight, chitosan can be classified as low, medium, and high molecular weight. Its molecular weight can be determined by HPLC and light diffusion but the viscometry method is the most common and simplest one [25]. In general, the molecular weight can be modified starting from a high molecular weight. Therefore, depolymerization methods are used to obtain low molecular weight edible chitosan films which more effective than high molecular weight in preserving papaya fruit and fish during their storage. The effect of the medium molecular weight of the polymer on the chitosan protein interaction was studied [26]. This study stated that increasing chitosan molecular weight from 15 to $200 \mathrm{kDa}$ increases the binding constant between bovine serum albumin and chitosan in complexes.

\subsubsection{Degree of deacetylation}

The degree of deacetylation (DD) is the ratio between 2-acetamido-2-deoxy- $\beta$-Dglucopyranose and 2-amino-2-deoxy- $\beta$-D-glucopyranose. When all the monomer units are 2amino-2-deoxy- $\beta$-D-glucopyranose, the biopolymer is completely deacetylated and DD is $100 \%$.

137 When the percentage of 2-amino-2-deoxy- $\beta$-D-glucopyranose units reaches $50 \%$ or more, the polymer being normally soluble in aqueous acid media is called chitosan [27]. In the same way, like MW, DD influences the properties and applications of chitosan. For example, an increase in

140 DD contributes to an increase in free amino groups in the chitosan chain. Therefore, the solubility and the polycation quality of chitosan also increase. The DD can be tested using spectroscopic methods such as Fourier transform infrared spectroscopy (FTIR), X-ray diffraction (XRD), and nuclear magnetic resonance (NMR) [28]. Chitosan with different DD can be obtained by varying 
144 the deacetylation reaction conditions. The adsorption of anionic methyl orange dye by using 145 chitosan with different degrees of deacetylation was reported. The results showed an increase in 146 DD to $84 \%$ directly proportional to the adsorption capacity from 266 to $373 \mathrm{mg} / \mathrm{g}$ [29]. Similar 147 findings were also obtained elsewhere for the adsorption of food dyes by chitosan in the binary 148 system [30]. The DD has been shown in the literature to influence the mechanical properties 149 (elongation and tensile strength) of chitosan films [31].

\subsubsection{Crystal structure}

The crystallinity of chitosan is the ratio between the crystalline and amorphous fractions of a biopolymer. This property is quantified as a crystallinity index (CI) which is determined by the relation between the characteristic peaks of X-ray diffraction (XRD). In the solid-state, chitosan is a semi-crystalline and polymorphic biopolymer, its unit cell is orthorhombic and contains two antiparallel chains free from water molecules [32]. Additionally, chitosan is found in three polymorphic phases that differ in the degree of crystallinity $(\alpha, \beta$, and $\gamma)$, depending on the source and arrangement of the chains. The commercial chitosan is a semi-crystalline polymer and CI is a function of DD. Also, the quantitative assessment of CI is important because this property affects the swelling, porosity, hydration, and absorption properties. Thakhiew's et al. [33] works showed that different methods of drying affect the physical and mechanical properties of chitosan films. They used hot air drying (HD) and low-pressure steam drying (LPSSD). They found that LPSSD provides films with a lower CI value (3-5\%) than films made from HD (10-16\%). As a result, the swelling degree was extended to 48-100\% (LSSPD) and 75-110\% (HD) [26, 34].

\subsubsection{Surface area and particle size features} associated with the porosity, pore-volume, and pore size distribution of the chitosan particles. Chitosan powder or flakes are known to have a reduced surface area (less than $10 \mathrm{~m}^{2} / \mathrm{g}$ ) being non-porous materials. Particles smaller than $1 \mathrm{~mm}$ is commonly used in most applications. The surface area and particle size are crucial for applications such as adsorption and immobilization of enzymes since many accessible sites and porous structures are required in these vital fields $[35,36]$. Some modifications have been made on chitosan to increase surface area because 172 naturally, it is a non-porous material. The surface area is usually determined by $\mathrm{N}_{2}$ adsorption- 
173 desorption isotherms by the Brunauer-Emmett-Teller (BET) method. Particle size can be 174 obtained using scanning electron microscopy (SEM), sieving test, or using a particle size analyzer. The effect of the particle size, surface area, and pore volume of chitosan on the adsorption of some food azo dyes FD\&C red 2 and FD\&C yellow 5 was studied before [37]. The particle sizes used were $0.10,0.18$, and $0.26 \mathrm{~mm}$ with surfaces of $4.2,3.4$, or $1.6 \mathrm{~m}^{2} / \mathrm{g}$. Accordingly, the results indicated that the adsorption potential is increased by an increase in surface area and a decrease in particle size.

\subsection{Properties of chitosan}

The properties of chitosan depend on its characteristics which are therefore responsible for the quality and extent of use of chitosan. Among the most important properties of chitosan are solubility, polycationic nature, film formation, antimicrobial activity, and biocompatibility [38, 39].

\subsubsection{Solubility and polycationic nature}

The solubility and polycationic nature of chitosan are fundamental properties to produce more derivatives and it is usually a strong base with primary amino groups that have a pKa of 6.3. Therefore, this biopolymer is soluble in acid solutions diluted to $\mathrm{pH}$ 6.0. At low $\mathrm{pH}$ values, the $\mathrm{H}^{+}$ions in solution protonate the $-\mathrm{NH}_{2}$ groups, which leads to the formation of $\left(\mathrm{NH}_{3}\right)^{+}$. Therefore, chitosan is converted into a polycationic biopolymer structure. On the other hand, when the $\mathrm{pH}$ is above 6.0, deprotonation occurs and chitosan becomes insoluble [40]. An insoluble-soluble transition occurs when $\mathrm{pKa}$ with a $\mathrm{pH}$ is between 6.0 and 6.5. Formic acid is the most suitable solvent, while acetic acid is used more often. Other alternatives are hydrochloric acid and nitric acid. Chitosan is insoluble in sulfuric and phosphoric acids as well as concentrated acids are not indicated since they can lead to depolymerization and deformation of the polymeric chains of chitosan [41]. The solubility of chitosan and chitosan-based materials depends on several important factors, including time, temperature, DD, MW, particle size, type, the concentration of acid, and reduction methods.

\subsubsection{Antimicrobial properties}


Chitosan provides antimicrobial activity observed in fungi, viruses, and bacteria (Gramnegative and gram-positive). This is a key property that allows it to be used in areas such as food storage, pharmacy, and biomedicine more than other materials [42-45]. Previous studies showed that the antibacterial property of chitosan is due to its polycationic nature. The polycationic chitosan molecule strongly interacts with the surface of the microbial cells, leading to something like a progressive shrinkage in the cell membrane and eventually to cell death. At the same concern, chitosan bactericidal activity is attributed to the electrostatic interaction between the $\left(\mathrm{NH}_{3}\right)^{+}$chitosan groups and phosphoryl groups of the cell membrane component, phospholipid [46]. In general, the polycationic nature of the chitosan molecule communicates with the components of the microorganism's anionic surface antigens (lipopolysaccharides and proteins), which contributes to the release of intracellular response: to fluctuations in the permeability barrier. Also, it prevents bacterial growth by stopping the entry of nutrients into the cell; once in the cell, binds to DNA and thus inhibits the synthesis of proteins and RNA [44, 47, 48].

In some cases, a modification of chitosan is beneficial for developing a nanoantibiotic system able to antagonize both opportunistic and obligatory pathogenic bacteria (Fig. 3). In this study, the authors developed functionalized chitosan with 3,5-dinitro salicylic acid and hooked in antibiotic linezolid. This combination is tough on Methicillin-Resistant Staphylococcus Aureus (MRSA) and other microbes [49].

\subsubsection{Biocompatibility}

Biocompatibility can even be categorized as "compatibility with living tissues or a living system because it is non-toxic, physiologically reactive and does not cause immune rejection".

221 One of the principal characteristics of chitosan is its biocompatibility which allows for the use of 222 chitosan in tissue engineering, wound dressing, bone regeneration, and other related areas [50223 53]. In vitro and in vivo assays have been used to evaluate the cytotoxicity, safety, and 224 biocompatibility of chitosan inside the body [54].

\section{1.4. Chitosan hydrogel}

Hydrogels can be represented as a 3-dimensional polymeric matrix composed of natural or synthetic materials that would have a high degree of flexibility depending on their high water content $[55,56]$. They can hold significant amounts of water and are distinguished by a 
consistency such as soft rubber-like living tissue, making them a suitable material for different utilization. For different applications, chitosan can be used as a hydrogel but the compressive strength, in this case, is fragile. Therefore, the combination of chitosan with other materials is usually used to produce hydrogels. Some methods of producing such hydrogel include radiation crosslinking technology, freeze-thawing, dissolution/lyophilization, and others [57]. The quality of the hydrogels is checked using SEM, FTIR, XRD, DSC / TGA in the same way as other chitosan-based composites. Also, depending on the application, specific features like swelling, elastic modulus, gel resistance, mechanical properties were previously studied. Cheng et al. [58], developed the heat-sensitive chitosan-based hydrogel as a local eye drug delivery system of latanoprost for the treatment of glaucoma. Hydrogel has been characterized based on its rheological properties, the release of drugs in vitro, its biocompatibility in vivo, and in vitro study. The release of latanoprost from the developed hydrogel was achieved and showed a prolonged release profile. In the case of the rabbit glaucoma model, the Triamcinolone-Induced Intraocular Pressure Elevation was significantly reduced over one week. In addition, a weekly topical dose of $250 \mu \mathrm{g} / \mathrm{ml}$ containing latanoprost hydrogel remained within normal limits until the end of the study period. The hydrogel has desirable characteristics which correlate to its delayed latanoprost release. This new composition of chitosan in the future could be a noninvasive alternative to traditional eye drops for long-term intraocular pressure control. Fan et al. [59] produced a chitosan /gelatin/ polyvinyl alcohol (PVA) hydrogel for wound dressings utility. The gelatin was blended in a combination of PVA and chitosan using radical initiator $\gamma$ radiation. Various experimental conditions were modified, and hydrogels were widely characterized. The Maximum tensile force was 2.2 MPa. All the hydrogels had a strong clotting effect which further indicated that the hydrogels' hemostatic effect was optimal. Hydrogels have demonstrated strong resilience to water $\mathrm{pH}$, swelling and evaporation rates.

\section{Chitosan nanoparticles and nanocapsules form} bioavailability of the drug when taken orally. They have a good mucoadhesive capacity, enhanced bioavailability, and dissolution rate of hydrophobic drugs. Besides, chitosan improves the stability of labile drugs, the bioavailability, and the controlled release of the drug, as the particles are beyond the nanoscale range to effectively overcome barriers and enhance 
permeability. However, this polysaccharide as a drug release system has some disadvantages Its

260 low solubility at physiological $\mathrm{pH}$ affects the intake and causes a pre-systemic metabolism with oral administration of drugs. The ability of chitosan alone to encapsulate water-insoluble drugs is also harmful because the loading efficiency is generally quite low. Various chitosan derivatives have been created and have been proven to be effective, especially when they are in nanoparticle form to remedy these deficiencies [60].

Polymeric nanoparticles are commonly defined as solid colloidal particles of between $10 \mathrm{~nm}$ and $1 \mu \mathrm{m}$ in size [61]. Nanoparticles are used as a method for the integration and storage of active substances in different fields and consist of macromolecular and/or molecular structures in which active substances such as drugs are dissolved, stored, encapsulated, or even adsorbed, or maintained on the external interface. Nanoparticles can be classified into two major groups based on their morphology: nanospheres, as a rule, are solid structures with a homogeneous matrix structure in which resources are distributed uniformly; and nanocapsules with a traditional hollow shell structure consisting of a polymer membrane and an internal core of the included drug [62].

Among the various types of innovations in nanotechnology that can be found in the nanoparticles and nanocapsules field, it is important to emphasize that both materials are the main target of the inclusion of active substances, have a high carrying capacity, and provide controlled release. The fundamental significance in the use of nanoparticles/nanocapsules in biotechnological applications instead of microparticle systems lies in their ability to increase the

278 absorption rate, boost bioavailability and ensure delivery of targeted drugs for cancer treatment and IV distribution systems, and so on [63]. These systems, as a means of drug release, ensure the targeted release of drugs to cells instead of nonspecific diffusion and improve the solubilization and release of poorly water-soluble drugs, ensure the delivery of two or more drugs together and protect the drugs from undesirable degradations [64].

\subsection{Chitosan nanoparticles and nanocapsules preparation methods}

284 A production method in which the chitosan particle size was reduced to a nanometer size was first described in 1994 by Ohya et al [65]. The processes used to produce nanoparticles can be divided into different categories: (i) physicochemical processes in which the nanoparticles are precipitated by using preformed polymers, with yield induced by emulsification-solvent 
evaporation, diffusion, or reverses salting-out, [25]; (ii) by in-situ chemical synthesis of macromolecules which lead to polymerizations or interfacial polycondensation reactions; and (iii) mechanical processes using high energy devices such as high-pressure homogenizers, ultrasonic devices, or wet high energy milling. The main methods for producing chitosan nanoparticles and nanocapsules are ionic gelation, emulsification and crosslinking, complexation with polyelectrolytes, self-assembly and drying processes. Some features of each method are listed below with emphasis on the main aspects of the processes.

\subsubsection{Ionic gelation}

Ionic gelatin is probably the most common method of producing chitosan-based nanoparticles. Chitosan is a cationic polysaccharide able to gel on contact with polyvalent polyanions as tripolyphosphate (TPP). This interaction led to the formation of electrostatic interactions between opposite charges of the components $[63,66]$. At acidic $\mathrm{pH}$, it can spontaneously transform into particles in the submicron size. This process seemed to be very interesting because reproducible nanoparticles can be obtained in a soft medium (aqueous medium) and avoid the use of organic solvents and high temperatures. Using chitosan TPP the nanoparticles were prepared by dissolving it in a diluted solution of glacial $-\mathrm{CH}_{3} \mathrm{COOH}$. The resulting solution was magnetically stirred at room temperature until completely dissolved. Therefore, the $\mathrm{pH}$ increased to the expected value by adding $\mathrm{NaOH}$. A TPP solution can be prepared in various concentrations by dissolving the TPP in high purity water. Both solutions are filtered through a small pore filter. Then, the TPP solution is added dropwise to the chitosan solution with constant stirring until it forms an opaque suspension, which indicates the formation of particles with average diameter controlled by the molar ratio of chitosan/TPP [67]. The mechanism of chitosan nanoparticles formation by ionic gelation using TPP as well as particle internal structure and surface topology are determined by the following parameters the degree of acetylation and MW of chitosan, intrinsic viscosity, concentration, and the molar ratio of $\mathrm{NH}_{3}{ }^{+} /$TPP [68]. Other factors may also influence the properties of the nanoparticles, such as operating temperature, stirring speed, and flow rate of TPP addition. This can affect particle size and polydispersity, as they appear to significantly reduce the amount of nanoparticle aggregation. Smaller particles can be isolated from larger particles by filtration and ultracentrifugation [69]. 
This method effectively captures small hydrophobic and hydrophilic molecules, as well as peptides, proteins, and genes. The formation of electrostatic interactions within the components of the matrix is considered to be the main retention mechanism of these biologically active compounds. In addition to TPP, other polyanions can be used for crosslinking, for example, the active molecule adenosine triphosphate (ATP), fucoidan, and sodium metabisulphite $\left(\mathrm{Na}_{2} \mathrm{~S}_{2} \mathrm{O}_{5}\right)$

322 [70]. Catechol-containing molecules such as hydrocaffeic acid have demonstrated the ability to 323 increase the mucoadhesion of various polymers, including chitosan. Chitosan/TPP hydrocaffeic 324 conjugated nanoparticles obtained by ionic gelation showed 6 times greater mucoadhesion in the rabbit's small intestine than the unmodified nanoparticles [71]. Besides, by the same technique, 326 chitosan/calcium pyrophosphate hybrid microflowers shape was prepared and efficiently applied 327 in dye removal and enzymatic catalysis (Fig. 4) [72].

\subsubsection{Polyelectrolyte complex (PEC)}

PEC's forming of chitosan nanoparticles is based on the ionic interaction of chitosan with negatively charged polymers. The process occurs when polymers are mixed with opposite charges and lead to self-assembly or the formation of strong reversible electrostatic bonds in selfassembly systems. These direct interactions between the polymer chains result in the formation of complex polyelectrolyte networks with proportional structures, thus avoiding the use of covalent crosslinking agents. In particular, these polymer network systems are commonly used as biocompatible hydrogels and are sensitive to changes in surrounded environmental conditions [73]. The emergence and stability of the PEC are based on several factors including the degree of ionization within each polyelectrolyte with an opposite charge, density of the charges present in the structure of each polyelectrolyte, concentration, and proportion of polyelectrolytes in the mixture, a sequence of the mixture and the type and location of ionic groups. In polymer chains, the stability is also correlated with the MW of the polyelectrolytes, the flexibility of the polymer chain as well as the temperature, ionic resistance, and $\mathrm{pH}$ of the reaction medium [74]. In the presence of a common solvent, the excess polyelectrolytes charges (positive or negative) are consolidated, and a non-stoichiometric complex is formed. In contrast, the stoichiometric polyelectrolytic complex contains equal quantities of each opposite charge, and the total charge in the resulting complex is zero. In the case of chitosan, various polyelectrolyte complexes can 
form when the positively charged surface of chitosan interacts with anionic biopolymers such as

347 sodium alginate, pectin, carboxymethylcellulose, chondroitin sulfate, dextran sulfate [75], etc.

\section{$348 \quad$ 2.1.3. Drying processes}

This process is frequently used in drugs, chemicals, cosmetics, materials, and food science technology. The first patent for this technology originated in the early 1870s. Even during the drying process, the water/solvents are separated through evaporation from liquids, solids, or semi-solids and the resulting vapor is collected by vacuum. Hot air, a microwave oven, spray drying, freezing, supercritical drying, and natural drying with air are common methods for drying. The techniques of spray drying and supercritical drying are often used in the production of chitosan particle systems because they are fast, light, continuous, reproducible, and adapt well to the active material. They are scaled-down in one phase without modification. These techniques are important and can achieve particles of various sizes and high stability [76]. Spray drying has been reported to be used to produce dry granules and powders from mixtures of drugs and excipients in the form of a solution or suspension. The versatility of this approach has been developed to include the synthesis of microparticles from various protein-filled polymeric materials, vaccine antigens, and therapies. Spray drying provides a comprehensive, convenient, one-step, and protein-friendly method for protein-loaded chitosan micro/nanoparticles [77]. Besides, it is widely used in compounds including heat-sensitive substances such as extracts, pigments, drugs, aromatic oils, and flavors in various types of conveyors, which lead to the formation of polymeric nanoparticles or microparticles as well as nanocomposites [78]. The fundamental theory of spray drying is dependent on the use of a flux of hot air to dry spray drops. This method requires the preparation of an aqueous chitosan solution that is sprayed into a drying chamber through a nozzle to achieve the desired particles. The resulting particles have a smooth surface, a spherical shape, and a reduced size distribution, and they also exhibit good drug stability with high efficiency when the drug is turned on [79]. Other benefits of this process

372 can increase stabilization and control the release of the drug. The size of the particles produced is 373 affected by the velocity of the sprayed flow, the degree of crosslinking, and the flow rate of the 374 airflow [80].

\subsubsection{Emulsion system (diffusion process of oil/water emulsion)}


Nanoparticles can be obtained using nanoemulsion in various ways. Emulsification processes usually require high mechanical energy to obtain small droplet sizes. The low power method should be considered according to the physical behavior of the system based on the mixing of oil and water in the presence of surfactants [81]. Firstly, a dispersion of chitosan in a glacial acetic acid solution is prepared as an aqueous component. Then, a drop of a hydrophobic solvent, such as cyclohexane, cyclohexanol, soybean oil, or another solvent with similar properties is added to the chitosan solution, all mixing continuously. The resulting mixture is treated with a surfactant, for example, Triton X-100 or Tween with constant stirring until the mixture becomes translucent, which indicates the formation of emulsion containing nanoparticles [82]. Another option is to pre-mix the surfactant in one of the solutions, saving a step in the process. In general, the particles formed in the emulsion system have nanocapsules structures, high drug loading efficiency, and better bioavailability [83]. ultrasound and an increase in the reaction temperature can collaborate with the process. There are various modifications in the preparation of an emulsion using water/ oil or oil in the water system, for example, the addition of gelling agents such as chlorides, TPP, or polycations is performed [84]. Although the emulsification method facilitates particle size control, strong crosslinking agents are used in this process and complete removal of unreacted crosslinking agents can be an issue [85].

\subsubsection{Self-organized nanoparticles}

Self-assembled nanoparticles can be formed in aqueous media of amphiphilic polymers. Hydrophobic interactions between amphiphilic polymer components tend to minimize interfacial energy and allow nanoparticle formation. Although chitosan is not an amphiphilic biopolymer, the self-organizing nanoparticles can be obtained by structural modification of chitosan to bind a compound such as a palmitoyl, which causes self-aggregation of chitosan molecules [86]. The hydrophobicity of chitosan is increased with the introduction of phthaloyl, polyester, fatty acids, 402 cholesterol [87].

Structural changes in chitosan derivatives can make it amphiphilic, resulting in nanoparticles self-assembling and be used as drug delivery vehicles. Besides, the quaternary chitosan 
derivatives were synthesized using (5-pentyl) trimethylammonium and dodecyl aldehyde. Autoaggregates formed in an aqueous solution have a dimensional distribution of 250 to $500 \mathrm{~nm}$ and have a hydrophobic core, which can be used as a carrier for hydrophobic substances [88].

\subsection{Chitosan nanoparticles and nanocapsules characterization techniques}

Dimensions and size distribution are important tools because of their ability to recognize the physicochemical and pharmaceutical behavior of the compounds present in the systems. Depending on the form of preparation, laser diffraction and a coulter counter can be used to determine particle size. However, the most common method is dynamic light scattering (DLS), which is used for measuring particle size distribution. The uniformity of the particle size is obtained by using polydispersity (PDI), with PDI values between 0.10 and 0.25 indicating a reduced dimensional distribution and higher values indicating a wide dimensional distribution [89]. Particles containing chitosan or other charged molecules show a zeta potential which corresponds to the electrical potential represented by the surface of the material in the solvent and is highly dependent on the particle's composition. The zeta potential uses the electrophoresis principle that reflects the electrical charges on the surface of the particles. Electrical fields are induced from scattered particles, whereby the particles move to the oppositely - charged electrode at a rate comparable to the zeta potential value. For a dispersion system, a high level of negative or positive zeta potential indicates the stability of the particles towards aggregation, which means a stable system. This parameter can be associated with particle stability and the zeta potential values less than $\pm 30 \mathrm{mV}$ contribute to low stability leading to a possible aggregation of the particles [90]. Various microscopy methods can be used to confirm particle size, to observe and characterize the morphology and structure of these nanoparticles. Atomic force microscopy (AFM) and SEM are some examples of devices used for this purpose, although transmission electron microscopy (TEM) is the most used method since provides information on the structure, shape, and actual particle size as well as an estimation of the wall thickness in capsules [91]. The structural modifications of chitosan were mainly characterized by FTIR, ${ }^{1} \mathrm{H}-\mathrm{NMR}$, and ${ }^{13} \mathrm{C}-\mathrm{NMR}$. These modifications are generally used to illustrate the physicochemical properties of the resulting nanoparticles, to detect interaction with active substances, and study how chitosan binds with specific cellular receptors [91]. 
The specific surface characteristics of the resulting particle system can be integrated by X-ray photoelectron spectroscopy (XPS). This technique provides details on the elements through which particles, chemical bonds, and extensive high sensitivity molecular information derive. Besides, XPS extends a detailed composition of the surface and the proximal surface chemistry analysis of the particles [92].

\subsection{Applications of chitosan nanoparticles and nanocapsules}

Nanosystems based on chitosan can be used in various fields of applications, depending on the system's preferred structure and chemical aspects of biopolymers, such as pKa, degree of deacetylation. Chitosan has two types of hydroxyl groups in its structure: the primary hydroxyl 443 group in the Carbon-6 position and the secondary hydroxyl group in the Carbon-3 position in the 444 glucosamine unit. Additionally, t Carbon-2 position works for a group $-\mathrm{NH}_{2}$. Therefore, the structure of chitosan can be manipulated and modified by inserting new functional groups or through cross-linking reactions, whereby the obtained derivatives are appropriated for the desired application. Chitosan nanoparticles modified with acetophenone by Schiff-based condensation were fabricated by ionic gelation in the presence of TPP as a gelling agent. The excellent adsorption capacity was observed towards various divalent metals: $\mathrm{Cu}^{2+}, \mathrm{Cd}^{2+}, \mathrm{Hg}^{2+,}$ and $\mathrm{Pb}^{2+}$ at $\mathrm{pH}$ 7.0. It was being used to eliminate these metal ions contaminating tap water, seawater, and industrial wastewater, as a result of which the adsorption balance is established within 30 minutes after the contact time between the absorbent and metal ions [93]. Also, the $100 \mathrm{~nm}$ chitosan nanoparticles can remove the hexavalent chromium $\left(\mathrm{Cr}^{6+}\right)$ from water, which requires the presence of TPP for the efficient adsorption of the metals. $\mathrm{Cr}^{6+}$ is adsorbed on the surface of chitosan/ TPP/nanoparticles and reduces $\mathrm{Cr}^{6+}$ to $\mathrm{Cr}^{3+}$, the reduced form of $\mathrm{Cr}^{3+}$ is less or nontoxic. Consequently, this system can be effectively used for detoxification and purification of water from $\mathrm{Cr}^{6+}$ [94]. $\mathrm{Co}^{2+}, \mathrm{Ni}^{2+}$ and $\mathrm{Cu}^{2+}$ can also be removed from water using modified chitosan nanoparticles by preparing chitosan -poly(methacrylate) using PEC technique with particle size from 100 to $120 \mathrm{~nm}$ [95]. Recently, modified chitosan nanoparticles were used to 460 eliminate $\mathrm{Cr}^{6+}$ and/or Rhodamine B (RHB) dye from a complex water system. About $98.4 \%$ of $\mathrm{Cr}^{6+}$ and $96.2 \%$ of RHB was adsorbed on the surface of modified chitosan nanoparticles. The mechanism was disused based on hydrogen bonding interaction, electrostatic attraction, and $\pi-\pi$ stacking force (Fig. 5). Accordingly, this nanoadsorbent system is preferential adsorbent 
464 compared to others reported materials dealing with $\mathrm{Cr}^{6+}$ and RHB pollutants owing to the 465 functionality of the unsubstituted amino group in the chitosan structure. Besides, green 466 microwave method was adopted in this paper which save cost, time, and required low energy for 467 complete the functionality process [96].

In another study, chitosan or starch were added to poly (vinyl alcohol) NPs (PVA $)$ as nano adsorbent for oil spills from wastewater [61]. The optimal adsorption amount $\left(\mathrm{q}_{\mathrm{g}} / \mathrm{g}\right)$ of PVAn blended chitosan was 33.6, 73.96, $93.1 \mathrm{~g} / \mathrm{g}$ for hydraulic oil, kerosene, and toluene oils, respectively. This study reveals the correlation between different types of oil and their adsorption 472 efficiency, and the mechanism was illustrated based on the diffusion mechanism. The incorporation of chitosan enhanced the reusability performance of the binary blend even after six cycles. This property produces adequate recoveries between 71.8 to $96.3 \%$ correlating to the high mechanical strength of the nanoblends which can be used efficiently in the practical application of oil spill recovery (Fig. 6).

In agriculture, chitosan nanoparticles have been used as herbicidal and pesticidal formulations of such compounds as paraquat, clomazone, and acetamiprid. Paraquat is a nonselective herbicide that is often used to control weeds. However, its solubility in water and soil absorption can cause toxicity in humans. This herbicide has been encapsulated in chitosan /alginate and chitosan/TPP nanoparticles produced by $\mathrm{Ca}(\mathrm{II})$ complexation [97] and by the ionic gelation technique [98], respectively, with an obtained diameter of about 635 and $300 \mathrm{~nm}$. These systems have shown less toxicity than a pure compound, which can reduce adverse effects on human health and the surrounded environment. Clomazone is a main component in rice fields and has been encapsulated in chitosan/alginate nanoparticles with a particle size of about $200 \mathrm{~nm}$ [99]. polyphenols extracted from various sources. The polyphenols in tea extract have been 493 successfully encapsulated with chitosan nanoparticles with an average size of 150-350 nm [101]. 
Chitosan is used in the nutrition and food industry to protect food or certain nutrients for human consumption via packaging and control of foodborne pathogens. The nisin-containing chitosan -carrageenan nanocapsules were obtained using the PEC method with the smallest particle size of approximately $500 \mathrm{~nm}$. This method was tested in vitro to assess the antimicrobial effects against Micrococcus luteus, Pseudomonas aeruginosa Salmonella enterica, and Enterobacter aerogenes, followed by an antibacterial activity test in tomato juice [102].

Small interfering RNA (siRNA) may be an effective candidate for disrupting genes in undesirable eukaryotic cells that are associated with diseases such as cancer, viral infections, and other genetic conditions. The siRNA consists of 21-23 base pairs that can induce a reduction in target genes [103]. Due to its positive natural properties, chitosan-based nanoparticles provide electrostatic interaction with negatively charged siRNA and thus improve their bioavailability. Therefore, siRNA is destructive for DNA and achieving a high level of safe gene silencing [104]. Studies conducted on a mouse model of ovarian cancer have led to significant transfection and in vivo accumulation of cells, leading to effective inhibition of uncontrolled proliferation in vivo. In this study, the nanoparticles-lactate of polyethylene glycol / folic acid chitosan oligosaccharide were obtained by ion gelation and showed an average size of about $200 \mathrm{~nm}$. Various other studies have shown that chitosan-based nanoparticles are useful for the encapsulation and release of siRNA [105]. The small interfering RNA was successfully incorporated by ionic gelation into the chitosan TPP with a particle size of 78-200 nm [106]. These systems are in vitro or in vivo tested against melanoma B16 cells [107]; Lung cancer [105]; Cardiomoblast cells [108]; breast cancer [109]. According to the drug delivery line established by chitosan and modified chitosan nanoparticles, the treatment of brain diseases (tumor diseases, neurodegenerative, cerebrovascular) in which drugs must penetrate the brain has been improved for these systems. Indeed most of the compounds do not reach the cerebral blood flow barrier due to the protection of the brain guaranteed by the regulation of the blood-brain barrier (BBB) [110]. The drugs were administered via the nasal passage to overcome the limitation imposed by the BBB. They settled in the respiratory epithelium, entered the system circulation and therefore the BBB could be 521 crossed [111].

Chitosan exhibits mucoadhesive properties, which have been carefully studied and improved 
524 drugs, such as a chitosan-coated lipid matrix containing lipophilic dye Nile Red and near-infrared 525 dye Dir [112]; Buspirone hydrochloride encapsulated in chitosan nanoparticles thiolate obtained 526 by ionic gelation (200-600 nm) [113]. The intranasal pathways using chitosan nanoparticles have 527 shown effective biocompatibility and biodistribution through the human bronchial epithelium 528 (16HBE14p) [114]. Also, chitosan nanoparticles have been used in various diseases, for example, anxiety disorders [115]; and Neuroprotection in Alzheimer's disease [116]. Further, the mucoadhesive property of chitosan may also be useful for the administration of drugs for controlled oral, nasal, ocular [84], vaginal [117] local, and transdermal administration [81].

The mechanism responsible for the penetration effect is based on its positive charges. 533 However, a more pronounced cationic property was obtained by trimethylation of the primary 534 amino group without improved permeability properties indicating a more complex mechanism 535 than expected [118]. High molecular weight and a high degree of deacetylation improve 536 epithelial permeability. PEGlation, N-succinylation, and thiolation can also significantly improve 537 their mucoadhesive properties. Thiolated chitosan forms disulfide bonds with glycoproteins in the 538 mucosa and the effect can be increased using mercaptonicotinamide [119].

\section{Chitosan nanofibers}

Nanofibrous scaffolds have attracted an intensive interest in the last years due to their 541 feasibility to be used for numerous applications including water filtration, wound healing, 542 dressing, and medical clothing [120]. It might be noticed that the obtained accompanied 543 properties of produced nanofibers are susceptible to the preparation conditions. These conditions 544 include the type of polymer, viscosity, injection rate, voltage, orientation, and even the humidity 545 [121]. The obtained properties might be changed upon variation of the former conditions 546 involving fibers' diameters, porosity content, and surface roughness. Therefore, the selection of 547 the polymeric phase with adequate conditions could lead to a formation of nanofibrous scaffolds 548 with appropriate behaviors. For instance, both fibers' diameters and content of porosity are the 549 main controlling factors of mechanical properties. However, using these scaffolds for tissue 550 engineering applications is preferred to be conducted with a highly porous surface. This is 551 because high interconnected porosity could introduce a simple way for vascularization besides 552 nutrients and oxygen transformation. Furthermore, keeping the moisture of injured skin could 
encourage fast healing; therefore, tailoring the properties of the fabricated electrospun nanofibrous scaffolds should meet the requirement of the suggested application.

It can be mentioned that chitosan has been used intensively for heavy metals removal from aqueous solutions and preferred rather than different nanocomposites in the removal efficiency [122-126]. The presence of amino and hydroxyl anions in chitosan structure might induce electrostatic forces towards the targeted ions, other physical interactions help for adsorption of ions through a porous surface. Otherwise, besides nanofibers, chitosan is produced in numerous shapes for various utilizations including balls, micro/nano-particles, or sponges[127].

\subsection{Production of chitosan nanofibers}

There are several methods for producing chitosan nanofibers, such as electrospinning forcing, blowing a solution, coaxial electrospinning, centrifugal electrospinning [128]. Herein, we introduce a comprehensive description of techniques used for the preparation of chitosan nanofibers and their applications in different fields of technology.

\subsubsection{Electrospinning}

Nanofibers might be produced via different techniques, however, one of the most facile and low costed ones is the electrospinning technique $[129,130]$. The technique is based on applying a high voltage on a polymeric solution to be elongated and sputtered on a moving/fixed substrate to produce nanofibers. The optimum behavior of electrospun nanofibers could be assigned to the

high surface to mass ratio ( $\left.40-100 \mathrm{~m}^{2} / \mathrm{g}\right)$, which can be controlled considerably [131].

The electrospun nanofibers configuration is established on a uniaxial tension for a viscoelastic gel. A typical electrical torsion formation contains three main components: a capillary tube gathered to a needle, a high voltage source, and a substrate for fibers' collector, which is illustrated in Fig. 7 [132].

It is obvious that in the electrospinning technique, a high electric field is required and applied over the distance between the syringe tip and the collection plate. The polymeric viscous gel is placed in a syringe and injected into a pump, which creates a steady flow. As soon as the electric field reaches a certain critical value that the repulsive electrostatic forces exceed the surface tension, the flow of charged fluid is expelled from the conical protrusion, which is known as the 
581 Taylor cone. Finally, the jet lengthens and breaks constantly, as a result of the nanofibrous 582 scaffolds are deposited on the collector with instantly fast solvent evaporation [133].

583 The important parameters in the electrospinning process are solution parameters and process parameters. The parameters of the solution are determined dependent on the characteristics of the solvent and the polymer, including type and concentration of the polymer, molecular weight, viscosity, surface tension, dielectric constant, and conductivity. The process parameters include the voltage, distance between the tip of the nozzle and collector, flow rate of the polymer solution, design and position of the nozzle, composition of the collector, geometry, and speed of rotation. Environmental parameters include temperature, humidity, and air velocity [134, 135].

Concerning solution parameters, the increase in polymer viscosity or concentration contributes to increased fiber diameter. However, if the viscosity of the polymer solution is too low, the ejected jet can decompose into droplets and lead to electrospray [136]. As a rule, low surface tension values lead to the formation of pearl less fibers, consequently, low stresses can be applied during electrospinning. Also, low conductivity polymer solutions cannot be welded, as there is no surface charge on a drop of liquid needed to form a Taylor cone. On the other hand, high electrical conductivity causes a decrease in the tangential electric field across the surface of 597 a drop of liquid thus inhibits Taylor's cones from forming. Electrospinning is a successful machine for generating controlled electric voltage on a polymer solution dissolved in a solvent with high dielectricity constants that led to the formation of thinner nanofibers [137]. Applying high voltage on the charged solution boosts the electrostatic force, which increases the jet's elongation, resulting in a thinner diameter of the fiber. Voltages between 5 and $40 \mathrm{kV}$ are generally applied during electrospinning according to most of the automatically established machines. A larger distance between the row and the collector can increase the elongation time due to the obtained thinner fiber length. Some high volatility solvents, high temperatures, and low humidity will support solvent evaporation and causing porous fiber surface [138]. However, in some cases some of non-polymer molecules can be electrospun without the use of polymer as a carrier [139].

Chitosan has proven to be a problem for the electrospun. Unfortunately, the polycationic 
611 by electroforming chitosan solutions in $90 \mathrm{wt}$ \% aqueous acetic acid or by using a certain solvent 612 such as trifluoroacetic acid (TFA), dichloromethane (DCM), and formic acid. However, traces of 613 solvent or toxic organic acid in the products are harmful, especially if applied to human skin or 614 injured tissues [140].

615 Recently, the nanofiber mats have been made of electrospun chitosan with biodegradable 616 synthetic polymers such as PVA, polyethylene oxide (PEO), poly (vinylpyrrolidone) (PVP), poly 617 (lactic acid) (PLA). Polycaprolactone (PCL) and polyethylene terephthalate (PET). The 618 biocompatible, mechanical, antimicrobial activity, and other properties of chitosan nanofibers 619 have been significantly improved with the addition of PVA, PLA, PCL, PEO, PET, and PVP 620 [141]. To improve the biological efficiency of chitosan-derived nanofibers, researchers sought to 621 squeeze chitosan using natural polymers such as collagen, gelatin, and elastin [142].

\subsubsection{Coaxial electrospinning}

623 Coaxial electrospinning is a modern and economical approach for producing coaxially 624 structured nanofibers. The typical coaxial configuration consists of two concentric capillaries 625 (two different solutions), the solutions of the core and the shell of which they come out and join 626 at the end, forming a core-shell nanofiber (Fig. 8). The key benefit of this approach is to 627 strengthen material that is less rotating using a highly rotating material [143].

As a result, various materials including inorganic and organic compounds have been successfully combined as functional nanocomposites for high added value. The technique has been widely used to control secondary fibrous structures, to encapsulate drugs or biological agents in fibers, to produce nanofibers from non-fibrous materials, and to incorporate functional 632 fluids into the fiber matrix. Various biologically active drugs and substances, such as DNA, 633 proteins, antibiotics, can be entrapped directly into the nucleus that is protected by the formation 634 of the upper layer [144].

It has been stated that some hard-to-process polymer solutions could be co-electrospun to create ultrafine cores in the shells of other polymer materials. Chitosan nanofibers have recently 637 been obtained by coaxial electrospinning. Core and coating nanofibers can be made of synthetic polymers with high spinning properties. Recently, produced chitosan nanofibers by coaxial 
640 $6413 \mathrm{ml} / \mathrm{hr}$. [145].

merging chitosan with PEO at the ratio of $80: 20$ shell to the core, voltage $25 \mathrm{kV}$ and the flow rate

\subsubsection{Characteristics of chitosan nanofibers}

Nanofibers have a large surface area, small pore sizes, and the ability to create threedimensional structures. Also, its chemical, physical, and biological properties attract attention in the field of materials science, mechanics, electronics, medicine, plastics, and energy. Therefore, characterization methods are needed to effectively track the size, morphology, and dimension of these nanomaterials to evaluate and develop their production process. Also, it is necessary to know the properties of nanofibers which determines whether the resulting material is suitable for a specific application, and some of these characterizations are shown in Table (1) [146].

\section{Some applications of chitosan nanofibers}

Chitosan nanofibers can be used in various fields mainly due to the presence of $-\mathrm{NH}_{2}$ and -OH groups, along with their structure. Some of the possible uses of chitosan nanofibers are in the areas of drug delivery, biosensor, food packaging, water purification, tissue engineering, and wound dressing. This biopolymeric nanofibrous offers enormous structural possibilities for chemical modifications that create new properties, particularly in the biomedical field.

\subsection{Purification and treatment of contaminated water}

The demand for high-quality water has increased in recent decades [96, 125, 147, 148]. Drinking contaminated water can cause millions of deaths every year, mainly due to pathogen contamination. Membrane filtration is a recognized technology for reducing the contamination of drinking water. Further, bacteria can be easily killed by the ultrafiltration membrane upon using antibacterial surfaces that were made up of silver ions and other metals [149]. Also, the viruses were removed through nanofiltration membranes according to the size exclusion principle. One of the materials used for filtration is chitosan nanofibers owing to their significant properties in wastewater treatment, agriculture, pharmaceuticals, food science, textiles, and biotechnology [150]. Recently, chitosan nanofibers have been extensively studied for water treatment. This nanofiber material is described as a natural polymer, which is suitable for the elimination of heavy metals and colored dyes from water. $\beta$-cyclodextrin/chitosan/PVA nanofiber webs were 
designed by electrospinning and utilized in the dynamic adsorption of organic micropollutants and heavy metal ions from water [151].

Surgutskaia et al. manufactured aqueous PEO/chitosan nanofibers to adsorb nickel $\left(\mathrm{Ni}^{2+}\right)$, cadmium $\left(\mathrm{Cd}^{2+}\right)$, lead $\left(\mathrm{Pb}^{2+}\right)$, and copper $\left(\mathrm{Cu}^{2+}\right)$ from a solution. The authors observed that the maximum adsorption capacity of $\mathrm{Ni}^{2+}, \mathrm{Cu}^{2+}, \mathrm{Cd}^{2+}$ and $\mathrm{Pb}^{2+}$ ions through a $\mathrm{PEO} /$ chitosan nanofiber membrane follow the order: $\mathrm{Ni}^{2+}>\mathrm{Cu}^{2+}>\mathrm{Cd}^{2+}>\mathrm{Pb}^{2+}$. The measured thermodynamic parameters, $\Delta \mathrm{G}^{\circ}, \Delta \mathrm{H}^{\circ}$, and $\Delta \mathrm{S}^{\circ}$ demonstrated that the adsorption of this system was feasible, spontaneous, and endothermic in nature. After five cycles of adsorption-desorption, the reusability was calculated and the results showed that the PEO/chitosan nanofibers can be widely used in the continuous adsorption process [152].

Recently, created chitosan nanofibers have been functionalized with $\mathrm{TiO}_{2}$ NPs to remove heavy metal ions without electrospinning [153]. The authors showed that $\mathrm{Au} @ \mathrm{TiO}_{2}$ was successfully prepared using the photo precipitation process and then loaded into a matrix of chitosan fibers, the resulting material showed plasmonic fibers effect. Photocatalytic and photoreduction efficiency were studied for many pollutants that were exposed to visible light irradiation. Plasmon fiber has a high decomposition efficiency compared to $\mathrm{Au} @ \mathrm{TiO}_{2}$.

\subsection{Wound dressing material}

The antibacterial resistance of microorganisms, as a rule, is one of the main problems in the field of wound care and their treatment, which leads to complications such as infections and delayed healing [154]. An appropriate dressing ought to be capable of absorbing exudates from the wound area and this allows water to evaporate at a certain point, preventing microbial transport [155]. Several studies are primarily developed innovative prophylactic antibiotics resistant microorganisms contaminated wounds. Titanium fibers of chitosan have been considered dressings because of their high efficiency against different bacterial strains [156].

Antimicrobial mats from colloidal nanofibers dispersions of AgNPs based on chitosan mixed with PVA for wound dressings were constructed by electrospinning [157]. This study showed that the PVA/ chitosan blend has a well antibactericidal activity against different bacterial strains. Also, the rotating webs made from the mixtures including AgNPs were better. Thus, the 
involvement of AgNPs in combination with PVA/ chitosan solutions has enhanced not only electrospinning efficiency but also the antibacterial ability of electrically shaped fibers sheet.

In a recent study, honey was added to the PVA/chitosan polymer matrix before electrospinning to evaluate the ability of these nanofibers to heal a wound. The results showed that PVA/chitosan/honey has a positive effect on the healing of burn wounds. This study reported that topical use of this bio fiber displayed significant healing activity in the burning region [158].

The capabilities of 3D printed chitosan for chronic wound healing utilization were used. The results displayed that after 24 exposure to the human fibroblasts cell line, more than $90 \%$ of cells still were viable, which demonstrated the high biocompatibility of these scaffolds and encourage their utilization for clinical applications [159]. Furthermore, N. M. Ergul et al. examined 3D printed polymeric blend containing chitosan/PVA encapsulated with hydroxyapatite (HAP) for tissue engineering. It was shown that grains size of HAP was around $1.21 \mu \mathrm{m}$, while the elasticity modulus of the fabricated scaffold reached around $91.14 \mathrm{MPa}$ for the ratios of chitosan/PVA/HAP (15 wt. \%) based on the added contents of HAP [160]. In addition, Min Zhu et al. studied the photoactivated antibacterial behavior of chitosan films doped with $\mathrm{Ag} / \mathrm{MoS}_{2}$. The results exhibited that antibacterial efficiency reached around $98.66 \%$ and $99.77 \%$ against $S$. aureus and E. coli, respectively under visible light after 20 minutes of continuous exposure [161]. Moreover, A.A. Menazea et al. investigated the effect of AuNPs embedded through a polymeric blend of chitosan/PVA for wound healing applications. They found that photoactivated antibacterial blend illustrated great potency towards both $E$. coli and $S$. aureus with inhibition zones of $13.1 \pm 1.3 \mathrm{~mm}$ and $14.8 \pm 2.4 \mathrm{~mm}$ for the highest additional AuNPs [162].

\subsection{Tissue engineering}

Polymer nanofibers are still of significant concern in regenerative medicine as building materials for restoring, preserving, or enhancing the function of human tissues damaged due to aging, sickness, injury, or birth defects. Tissue engineering can also be segregated into several segments depending on the tissue/organ class such as bone, ligaments, cartilage, tendons, liver, nerves, and skin [163]. There are some widely accepted basic requirements for the construction of polymer forests. The structure must have high porosity with the adequate distribution of pore sizes, a large surface, biodegradability, adequate mechanical properties, and biocompatibility. 
725 Various biocompatible polymers have been developed from natural materials such as collagen, 726 elastin, laminin, etc. [164].

727 Poly $(\varepsilon$-caprolactone $) /$ chitosan nanofibrous was fabricated using an electrospinning 728 technique to create bone and skin tissue [165]. The presence of chitosan in the chitosan /PCL 729 structure led to a significant improvement in hydrophilicity. Further, the cytocompatibility of the 730 chitosan /PCL framework was studied and proved to be non-toxic. All these results showed that 731 the chitosan /PCL nanofiber structure would be an excellent system for bones and skin tissue 732 engineering.

733 Another example produced chitosan /PVA fibers with $\mathrm{CaCO}_{3}$ incorporation. The authors 734 observed that a tensile test of fibers reinforced with $\mathrm{CaCO}_{3}$ at content $4 \%$ by weight gave an 735 elastic modulus sixty times higher than the value of the fibers without $\mathrm{CaCO}_{3}$. The 736 biocompatibility of the $\mathrm{CaCO}_{3}$-loaded fibers was also tested in a 14-day incubation with 737 simulated biological fluid (SBF) to induce the formation of apatite minerals and in a 14-day 738 analysis of cell proliferation with ATDC5 cells. Chitosan/PVA- $\mathrm{CaCO}_{3}$ is perhaps the most 739 effective medium for cell growth as compared to chitosan/PVA and chitosan/PVA-apatite. $740 \mathrm{CaCO}_{3}$-enhanced chitosan / PVA can be a viable substitute for cartilage [166].

\section{$741 \quad$ 4.4. Delivery of drugs}

742 Owing to large specific surface area nanofibers can deliver platforms for drugs, peptides, 743 and vaccine antigens. The release of the drug may be immediate, delayed, or modified depending 744 on the type of interaction between the polymer with the drug [167]. Usually, an immediate 745 release is noticed when a composition of a water-soluble substance and a water-soluble polymer 746 is used. However, the extended-release profile can be tailored by integrating the drug into other 747 nanocarriers, such as nanoparticles, liposomes, dendrimers, then loaded into nanofibers or use 748 hydrophobic polymers [168].

Chitosan nanofibers have recently exhibited controlled drug release affinity [169]. This 750 material may be useful for the delivery of proteins/peptides, anti-inflammatory drugs, antibiotics, 751 imaging agents. Fabrication of poly (d, l-lactide-glycolide) (PLGA)/ chitosan nanofiber structure 752 by electrospinning was also assessed [170]. The results of this study showed that the drug release 753 rate increased with increasing chitosan content, as the addition of chitosan improved 
754 hydrophilicity. The release rate from an aligned PLGA nanofiber skeleton was also lower than 755 that of a randomly oriented PLGA/ chitosan nanofiber skeleton.

756 Gold nanorods (AuNRs) containing double-fiber hybrid PVA/ chitosan nanofibers were 757 prepared using a simple electrical conjugation process. The authors found how these nanofibers are used as carriers for the doxorubicin anticancer (DOX) drug that is inserted into the nucleus of the cells. The integrated AuNRs and DOX in fibers effectively inhibit the growth and proliferation of ovarian cancer cells and, thanks to their unique optical properties, can also be used as a cell imaging medium. The nanofiber matrix, which integrates two roles of cell imaging and the delivery of drugs may have great potential for use in biomedical fields [171].

\section{3D chitosan printable scaffold}

\subsection{Chitosan printed in cartilage and bone regenerative medicine}

The architecture of chitosan and its stability in physiological environments are very analogous to that of glycosaminoglycan. Furthermore, chitosan degradation products are often elements of cartilage synthesis. These advantages of chitosan are widely used in the construction of bones and cartilages. Also, a coating of chitosan on the PCL scaffold contributed to better fixation and penetration of the cells. It is also recommended the chitosan to be mixed with other materials, such as biological glass and collagen, to improve the chemical, osteoconductive and mechanical properties of chitosan [172]. In a previous study, a new rapid prototyping system was developed for the delivery of robots to attain chitosan/HAP structures [173]. They added the HAP/ chitosan base to improve the osteoconductivity and mechanical strength of the scaffolds. In their analysis, they employed acetic acid to create chitosan hydrogel and HAP chitosan solution, supported by extrusion to obtain a gelatinous precipitate in a bath mixture of sodium hydroxide and ethanol. The increased use of sodium hydroxide led to the rapid deposition and poor mobility of the chitosan molecules at the edges of the contact structure. Conversely, a very low concentration of sodium hydroxide reduces the deposition rate and causes poor dimensional stability. Tests on cell culture showed acceptable results as well. Rapid prototype robotic delivery (RPBOD) may therefore be an effective method for producing chitosan-based structures.

Ascertaining printing parameters such as outlet pressure, exit speed, and initial exit height was a problem in a study by Ang et al. [173]. These parameters depend on the solution's 
783

784

785

786

787

788

789

790

791

792

793

794

795

796

797

798

799

800

801

802

803

804

805

806

807

808

809

810

811

812

viscosity; therefore, they differed for each composition and concentration. The macroscopic analysis, such as visual inspection, was used to solve this problem and establish coherent and uniform filaments. It is difficult to select the right binding to 3D print. It has been documented that different acids have been studied, such as citric acid, phosphoric acid, lactic acid, and sulphuric acid [174]. It was found that a shorter curing period was obtained with $20-40$ wt. $\%$ of binding citric acid, and the 30-40 wt.\% lactic acid [175].

Several chitosan binders were tested for 3D printing and obtained nearly identical results for lactic acid. They were double-checked using lactic acid, citric acid, and acetic acid as the binder by printing a similar composition (chitosan/HAP). They found the $40 \mathrm{wt}$. \% lactic acid boosts medium viscosity, fast solidification time, and high wettability. Therefore, lactic acid has the correct structure relative to other binders.

There was an improvement in structural stability with a ratio of $20 \mathrm{wt}$. \% of chitosan and 40 wt. $\%$ of binder lactic acid and after processing $40 \mathrm{wt}$. \% of lactic acid. The three-dimensional printing structures, which consist of 25 wt. \% of chitosan and 75 wt. \% of HAP is shown in Fig. 9. The resulting printed samples showed a compressive strength of $16.32 \mathrm{MPa}$, an elastic modulus of $4.4 \mathrm{GPa}$, and a porosity of $37.1 \%$. The low porosity is due to the destruction of the open pores due to the high concentration of lactic acid. They thought that the chitosan chemical structure allows attractive bio-stimulation. Their findings suggested that the elevated chitosan /HAP structure is a promising alternative to the bone regeneration process.

In another study, the mechanical property and cytocompatibility of a hybrid constitute synthetic polymer (polyethylene glycol diacrylate (PEGDA)) and chitosan was evaluated [176]. This study showed that chitosan molecular weight and the feeding ratio are effective parameters in the shaping and precision of the structure. The ratio of PEGDA to nutrition with chitosan was set at 5:1 and 10:1, which was the optimal range. Low chitosan molecular weight requires an excessive concentration of sodium acetate due to its higher deacetylation to neutralize its protonated amino groups. They used Stereolithography (SLA) to create frames, while a 7.5:1 ratio showed a boost printability effect. SLA can monitor the frame composition and porous structure, depending on the anatomical requirements of the patient. They designed bezels in an ear-shaped form with a laser beam of $405 \mathrm{~nm}$. The presence of strengthened chitosan in the resin increases the coefficient of swelling. Adequate mechanical properties and enormous potential for 
813 cell attachment contributed to the growth of an innovative design for the cartilage tissue 814 engineering field with complex geometry.

815 Also, chitosan /alginate was chosen as the desired combination because of its similarity to 816 natural cartilage glycosaminoglycans (GAGs) such as hyaluronic acid, keratin sulfate, and 817 chondroitin sulfate. The compositional form can give a desirable property such as compressive 818 strength or swelling potential. Besides, a neutral $\mathrm{pH}$ skeletal preparation provides a green 819 environment that guards the combined growth factor for a drug against denaturation. They had to 820 use a modern manufacturing process, integrating 3D printing with directional freezing to monitor 821 building micro- and macro-channels. The macro channels were generated using 3D printing of 822 positive and negative shapes in the methodology used, and the microchannels were developed by 823 spatial freezing of the solution. These scaffolds with simultaneous lamellar capillaries and 824 uprights made the cartilage quite normal for replication and showed better absorption of 825 inflammation. Besides, better distribution of cells together with the height and appropriate 826 diameter of the scaffolds also made a significant contribution to the process of regeneration 827 [177]. On the other hand, chitosan was mixed with gelatin without the need for further 828 treatments. This solution was combined with $\beta$-glycerophosphate forming hydrogel at the 829 physiological temperature and enhanced the cellular interaction of chitosan. This solution was 830 supplemented with hydrochloric acid and deionized water. Cells were introduced to the solution, 831 and a method of bioprinting was carried out. Their findings showed that solid contamination, 832 accumulation, and gelation during the 3D bioprinting can escalate to needle clogging, which can 833 be prevented by cooling the prepared solution; centrifugation and degassing under vacuum 834 prevent these problems during printing. They proved that even an increase in chitosan 835 concentration results in a greater viscosity and an intensified gelation time which reduces fiber 836 size. Furthermore, the distance between the needle and the impressed surface is an important 837 factor to use agarose gel in this space. The diameter of the fiber decreased with the increasing 838 speed of advance. The new bioprinter offers a simple and inexpensive way to print bi-substrates 839 of chitosan /gelatin and at the same time maintains high cell viability [178].

It was observed that these new low stability chitosan hydrogels are easily dissolved, as 841 mentioned in some cases in a previous study [179]. A new gelatin/sodium 842 alginate/carboxymethyl hydroxycarbonate- chitosan composition filled with bone marrow stem 
843 cells for three-dimensional bioprinting was reported. The addition of chitosan improves 844 proliferation, cell adhesion, and mechanical force owing to the ionic bond between the $-\mathrm{NH}_{2}$ 845 group of chitosan and the $-\mathrm{COOH}$ group of the alginate. To enhance water solubility, chitosan 846 was modified into carboxymethyl chitosan by dissolving gelatin in double distilled water, then 847 alginate and carboxymethyl chitosan subsequently added to the solution and the ions were crosslinked with $2 \% \mathrm{CaCl}_{2}$. The stem cells of the bone marrow were loaded into a hydrogel and a three-dimensional bioprint was performed. The mechanical feed system has been set to a speed range of 1 to $10 \mathrm{~mm}$ and a pressure range of 20 to 60 psi. They showed that the decomposition rate of hydrogels with carboxymethyl chitosan has decreased compared with hydrogels without chitosan, which means that carboxymethyl chitosan has improved the stability of the hydrogel.

853 Furthermore, the antibacterial activity with carboxymethyl chitosan has been improved compared to the hydrogels without chitosan.

Chitosan was modified for 3D bioprinting of artificial bone tissue in a previous study [180]. In this study, chitosan was modified to $\mathrm{N}$, O-carboxymethyl chitosan to react with the polyphosphate and improve the biologically active properties of the hydrogel. The N, Ocarboxymethyl chitosan was dissolved in physiological solution and added polyphosphate to the solution, followed by centrifugation to remove a gas bubble. The solution was mixed with an alginate aqueous solution and then, bioprinted using a 3D bio plotter. Next, Ca was added for networking and the hardening of bio-printing structures. The proposed composition induced SaOS-2 bone cells for biomineralization. These scaffolds for bioprinting were in vivo studied and demonstrated significant activity of regeneration induction in lower leg defects of rats. This composition seems a very productive material for the custom design of frames in the bones. with polycaprolactone. This combination shows desirable mechanical degradation and superior biological properties [165]. 
Recently, alginate and chitosan hydrogels with different content have been used to print 872 channels with a wall thickness of less than 200 microns, in which a robotic printer directly 873 printed microfluidic channels in the shape of a vessel tube. 1.0 M sodium hydroxide was used as 874 the crosslinking agent of chitosan and acetic acid to dissolve chitosan. The high content of 875 chitosan solution (4\%) required higher pressure, which led to an increase in shear stress and a 876 decrease in cell viability. On the contrary, the mechanical behavior is decreased at low chitosan 877 content (2\%). Therefore, chitosan with a concentration of 2.5 to $3 \%$ was chosen as the optimal 878 concentration range. On the other hand, they have shown that the distribution of polymers or the rate of crosslinking affects the diameter of the channels.

Higher delivery speeds obtained by extrusion led to thicker walls and build wider channels. The printed alginate microfluidic channels were continual and more homogeneous based on their findings, but the printed chitosan samples were more fragile and easily breakable. Even though the concentration and delivery speed of selected materials are effective factors during microchannel printing. Hence, the viability of the cells is increased in the form of a single channel or mass incorporated in the hydrogel and this process is considered a promising production route for the development of the vascular network [181].

\subsection{Printed chitosan in skin regeneration} recruitment of polymorphonuclear leukocytes or neutrophils in the wound area, fibroblast proliferation, hyaluronic acid synthesis, angiogenesis, and deposition of collagen. It contributes to accelerating the process of wound healing and preventing scar formation. The fragile attitude of chitosan in skin tissue engineering can sometimes be also rectified by mixing with collagen, 894 alginate, or other polymers [182].

\subsection{Cast chitosan for tissue engineering applications}

Hard tissues in mammalians consist of two main constituents: hydroxyapatite (HAP) as a mineral part and collagen fibers as the biological contributor. It might be reported that HAP seems to be configured in nanorods with diameters in the range of 5-10 $\mathrm{nm}$ and length about 30$50 \mathrm{~nm}$. Furthermore, these rods are embedded vertically through the nanofibers of collagen. It 
could be concluded that while HAP represents the predominant component to resist compression

901 stresses, collagen fibrous contribute mainly to deal with tensile stresses. In other words, the great 902 mechanical properties of hard tissues could be assigned to their hierarchical structure. Therefore, 903 a design of bone possesses the upper hand in mechanical properties than the types of constituents. 904 Besides, HAP is a distinct inorganic agent with a hexagonal structure. Besides its high 905 biocompatibility, bioactivity, low cytotoxicity, and osteoconductivity, it possesses numerous 906 crystallographic sites that are able to be substituted partially whereas crystal structure is 907 remaining. The biological HAP is usually substituted with versatile ions with different contents 908 such as carbonate which comes from the ambient atmosphere. Therefore, mimicking of hard 909 tissues in mammalians might be reached via designing a multi-doped HAP to be embedded 910 through a bio-polymeric agent, which could introduce adequate mechanical behaviors and avoid annealing procedures. In this issue, $\mathrm{Mg} / \mathrm{Ag}$ co-doped HAP was encapsulated into chitosan using a casted technique. The morphological features of the obtained scaffolds were shown in Fig. 10 (af) [183].

\section{Advantages, drawbacks, and future trends of chitosan 3D printing} biomolecules accurately on scaffolds. The production control facilitates the approximation of natural textiles with customized biological and mechanical properties suitable for repairing tissues [184]. Though 3D printing was a breakthrough in the development of scaffolding, it still represents a long way to organ reproduction. One of 3D printing's most significant problems is the development of a functional organ filled with vascular lumens of different sizes. All cell types need a network of vessels to gain access to nutrients and oxygen, as well as to expel waste. 922 Therefore, the creation of a complete and complex network of vascularization in the printed structure remains a mystery [185]. However, 3D printing technology has more possibilities than traditional scaffolding methods for developing the boundaries of the vascular system by checking 925 the parameters. Interestingly, the impression of the native organ has many problems. Biological imitation of the whole organ, considering its various types of cells and microstructures, is a rigorous procedure that requires great effort.

Up to date, there is an important issue that should be taken into consideration which is the economic factor for the preparation of 3D printed chitosan, in addition to the preparation 
930 drawbacks such as the huge time of the crosslinking process and poor mechanical features. Such 931 issues limit the utilization of chitosan 3D printing for most medical purposes (tissue engineering). 932 These issues can be avoided via adding new substances such as gelatin compounds which can act 933 as mediators for the creation of new functional groups [186]. Based on that, such gelatin has been 934 added to enhance the printability of chitosan. Additionally, gelation has been used to enhance the 935 thermal stability of chitosan decomposition occurred around $220^{\circ} \mathrm{C}$. The amazing features of 936 modified chitosan facilitate its application in many biomedical purposes due to its proper 937 biocompatibility and adhesion with the cells. Therefore, it is necessary to explore the most 938 parameters and materials that can be used for changing the undesired features of chitosan [187]. 939 Furthermore, the added compounds represent an important factor that affects the shape and 940 precision of the structure of interest. It has been reported that chitosan with low molecular weight 941 is more favorable than he chitosan with high molecular weight. To get high properties, chitosan 942 with low molecular weight should be then used. Moreover, chitosan with high molecular weight 943 forms a rigid crystalline structure owing to the presence of huge inter and intramolecular 944 hydrogen bonds along with its structure, which in turn, restricts its application in SLA 945 bioprinting. Besides, acetic acid which is used for dissolving chitosan can be used to overcome 946 partially the formation of free radicals. In a short, in SLA of chitosan, the photoinitiator 947 concentration is a very important aspect. It has been reported that the utilization of photoinitiator 948 has a threshold value. Above the threshold value, the photoinitiator can lead to poor cell adhesion 949 and cell viability [180]. Based on the aforementioned findings, it was observed that the 950 concentration of both solvent and materials is a very important factor affecting the mold structure 951 in terms of mechanical features and stability. Moreover, different parameters such as physical or 952 chemical agent impacts on the process and the crosslinking agent have been also checked, in turn, 953 leading to an increment in the diameter of both core and channels. Thus, 3D printing produced 954 chitosan with high viscosity and the presence of crosslinking agents with high concentration can 955 create drawbacks such as limiting the feeding, transportation of oxygen, and cell migration. For 956 that, viscosity and crosslinking concentration are highly appreciable for the formation of good 957 chitosan 3D printing [188]. On contrary, it is clarified that 3D chitosan can swell after the 958 printing process leading to decreasing the diameter and struts of the pores. Thus, for decreasing 959 the swelling factor of 3D chitosan, a crosslinking agent should be added in a high concentration 960 [189]. 
Previously, there is much-published work documented the development of a superior method (solvent-free) such as laser absorption of two photons and single-photon during shear deformation for the generating of chitosan with various molecular weights and allyl substituted derivatives scaffolds. The advantage of using such a method concerns the avoidance of organic and aqueous solvents with no problem associated with viscosities and distributors [190].

As previously mentioned, the evaluation of cytotoxicity for 3D chitosan illustrated the viability of cells and cell growth formation. However, there is a lack of studies about other factors that should be extensively examined. Some of these factors are biodegradability, absorption of water, and mechanical features of the formed 3D prints. All these categories will be discussed in detail in future studies. Moving to our discussion about the amazing properties of 3D printing structures; such morphological structure is a very important issue that should be accompanied by the prepared scaffolds that affect cellular behaviors. The 3D printing is more favorable to be prepared with a morphological structure containing huge porous structures with small sizes to be efficient during the penetration of cells. It has been documented in previous studies that 3D printed chitosan has been prepared with a pore size of less than $250 \mu \mathrm{m}$. Indeed, chitosan with a positive charge is more desired for cartilage tissue engineering domains [191].

The rapid gelation of a negatively charged polyanion in an aqueous medium acted as an inhibitor of the diffusion of various parts of bioprinting. As in the study which reported that chitosan has a sufficient gelling time, acceptable for 3D bioprinting. Besides, it has maximum printing precision, long life, and is the cheapest gel compared to other approved materials used before in the materials for clinical purposes. Although bioprinting skin tissues developed with chitosan and chitosan /collagen have not received FDA approval. Given the similarity of composites with other approved materials, the regulatory approval may be relatively simple in the future [192].

It could be observed that upon variation of compositional contributions, the surface morphology has been changed considerably. HAP with dimensions of $4 \mu \mathrm{m}$ has varied to be 1.25 -

$9875 \mu \mathrm{m}$ upon the changing of dopant concentrations. Furthermore, HAP grain was scattered through 988 chitosan fiber which has diameters in the range of 2.5-5 $\mu \mathrm{m}$. The compressive strength was 989 conducted and displayed that additional Mg (II) might introduce high resistance to compressive stresses. The optimum compressive value was achieved at around 15.0 MPa [183]. 
The materials based on chitosan have several technological applications that have already 993 been investigated and many others that are possible. For an effective investigation of chitosan994 based materials, it must be considered that all areas of applications depend on the properties of 995 the biopolymer. The properties, in turn, depend on the production process, which depends on the 996 raw materials. Therefore, a series of clearly defined steps must be followed by the raw material to 997 produce chitosan. Also, chitosan is easily modified in various ways to obtain special properties 998 that are used in different trends. Traditional and advanced analytical methods should be used in 999 all these phases to verify the main results. Besides, in this review, the chitosan nanofibers are 1000 limited owing to the cationic property and solubility of chitosan in acids and this also was 1001 discussed. To evaluate and develop the chitosan nanofibers production process, it is necessary to 1002 analyze its morphology, crystallinity, chemical composition, structural changes, and mechanical 1003 1004 1005 1006 properties. Since chitosan is a natural polymer with useful biological properties, its nanofibers hold promise for a variety of applications. The presence of $-\mathrm{NH}_{2}$ and $-\mathrm{OH}$ groups in chitosan nanofibers allows their use in fields such as biotechnology, wastewater treatment, medicine, and pharmaceuticals.

\section{Declaration of Competing Interest}

1008 All authors declare that there is no conflict of interest.

1010 References

1011 [1] G. Crini, Historical review on chitin and chitosan biopolymers, Environmental Chemistry 1012 Letters (2019) 1-21.

1013 [2] S.A. Kulkarni, V. Dharini, S.P. Selvam, M.M. Kumar, E.R. Sadiku, J. Jayaramudu, U.N. 1014 Gupta, Fabrication of Bionanocomposites from Chitin, Chitin-and Chitosan-Based 1015 Biocomposites for Food Packaging Applications, CRC Press2020, pp. 11-21.

1016 [3] M. Kumar, V. Vivekanand, N. Pareek, Insect Chitin and Chitosan: Structure, Properties, 1017 Production, and Implementation Prospective, Natural Materials and Products from Insects: 1018 Chemistry and Applications, Springer2020, pp. 51-66. 
1019 [4] Q. Luo, Y. Wang, Q. Han, L. Ji, H. Zhang, Z. Fei, Y. Wang, Comparison of the 1020 physicochemical, rheological, and morphologic properties of chitosan from four insects, 1021 Carbohydrate polymers 209 (2019) 266-275.

1022 [5] M.M. Fouda, M. El-Aassar, S.S. Al-Deyab, Antimicrobial activity of carboxymethyl 1023 chitosan/polyethylene oxide nanofibers embedded silver nanoparticles, Carbohydrate polymers 1024 92(2) (2013) 1012-1017.

1025 [6] K. Shoueir, H. El-Sheshtawy, M. Misbah, H. El-Hosainy, I. El-Mehasseb, M. El-Kemary, 1026 Fenton-like nanocatalyst for photodegradation of methylene blue under visible light activated by 1027 1028 1029 1030 1031 1032 1033 1034 1035 1036 1037 1038 1039 1040 1041 1042 1043 1044 1045 1046 hybrid green DNSA@Chitosan@ $\mathrm{MnFe}_{2} \mathrm{O}_{4}$, Carbohydrate polymers 197 (2018) 17-28.

[7] R.M. Abdel-Rahman, R. Hrdina, A. Abdel-Mohsen, M.M. Fouda, A. Soliman, F. Mohamed, K. Mohsin, T.D. Pinto, Chitin and chitosan from Brazilian Atlantic Coast: Isolation, characterization and antibacterial activity, International journal of biological macromolecules 80 (2015) 107-120.

[8] N. Elsayed, A. Alatawi, M. Monier, Diacetylmonoxine modified chitosan derived ionimprinted polymer for selective solid-phase extraction of nickel (II) ions, Reactive and Functional Polymers 151 (2020) 104570.

[9] J. Sakwanichol, S. Sungthongjeen, S. Puttipipatkhachorn, Preparation and characterization of chitosan aqueous dispersion as a pharmaceutical film forming material, Journal of Drug Delivery Science and Technology 54 (2019) 101230.

[10] B.A. Omogbai, M. Ikenebomeh, Solid-state fermentative production and bioactivity of fungal chitosan, Journal of Microbiology, Biotechnology and Food Sciences 2019 (2019) 172175.

[11] Y.N. Tan, P.P. Lee, W.N. Chen, Dual Extraction of Crustacean and Fungal Chitosan from a Single Mucor circinelloides Fermentation, Fermentation 6(2) (2020) 40.

[12] R. Mulyani, D. Mulyadi, N. Yusuf, Chitosan Membrane from Shrimp Shells (Panaeus modonon) as an Antibacterial Food, Journal of Physics: Conference Series, 2020, p. 072006.

[13] H.E. Knidri, J. Dahmani, A. Addaou, A. Laajeb, A. Lahsini, Rapid and efficient extraction of chitin and chitosan for scale-up production: Effect of process parameters on deacetylation degree and molecular weight, International journal of biological macromolecules 139 (2019) 1092-1102. 
1049 [14] P. Zhou, J. Li, T. Yan, X. Wang, J. Huang, Z. Kuang, M. Ye, M. Pan, Selectivity of 1050 deproteinization and demineralization using natural deep eutectic solvents for production of 1051 insect chitin (Hermetia illucens), Carbohydrate polymers 225 (2019) 115255.

1052 [15] M.-N. Marzieh, F. Zahra, E. Tahereh, K.-N. Sara, Comparison of the physicochemical and 1053 structural characteristics of enzymatic produced chitin and commercial chitin, International 1054 journal of biological macromolecules 139 (2019) 270-276.

1055 [16] A. Serrero, R. Vestberg, S. Montanari, Implant comprising oxidized cellulose and method 1056 for preparing such an implant, Google Patents, 2019.

1057 [17] D. Tahtat, H.H. Boutrig, A.N. Khodja, S. Benamer, Y. Hammache, M. Mahlous, The 1058 synergistic effect of gamma irradiation and alkaline soaking at low temperature on the pre1059 deacetylation of $\alpha$-chitin: Optimization by design of experiment, Carbohydrate polymers 215 1060 (2019) 39-46.

1061 [18] M.S. Yusharani, I. Ulfin, Y.L. Ni'mah, Synthesis of water-soluble chitosan from squid pens 1062 waste as raw material for capsule shell: Temperature deacetylation and reaction time, IOP 1063 Conference Series: Materials Science and Engineering, IOP Publishing, 2019, p. 012070.

1064 [19] E.A. Azmy, H.E. Hashem, E.A. Mohamed, N.A. Negm, Synthesis, characterization, 1065 swelling and antimicrobial efficacies of chemically modified chitosan biopolymer, Journal of 1066 Molecular Liquids 284 (2019) 748-754.

1067 [20] I. Lipatova, N. Losev, L. Makarova, The influence of the combined impact of shear stress 1068 and cavitation on the structure and sorption properties of chitin, Carbohydrate polymers 209 1069 (2019) 320-327.

1070 [21] T. Hahn, A. Roth, R. Ji, E. Schmitt, S. Zibek, Chitosan production with larval exoskeletons 1071 derived from the insect protein production, Journal of Biotechnology 310 (2020) 62-67.

1072 [22] L. Huang, S. Bi, J. Pang, M. Sun, C. Feng, X. Chen, Preparation and characterization of 1073 chitosan from crab shell (Portunus trituberculatus) by $\mathrm{NaOH} / \mathrm{urea}$ solution freeze-thaw 1074 pretreatment procedure, International Journal of Biological Macromolecules 147 (2020) 931-936.

1075 [23] D. Sugiyanti, P. Darmadji, S. Anggrahini, C. Anwar, U. Santoso, Preparation and 1076 characterization of chitosan from Indonesian Tambak Lorok shrimp shell waste and crab shell 1077 waste, Pakistan J. Nutr 17 (2018) 446-453. 
1078 [24] Y. Zhong, C. Zhuang, W. Gu, Y. Zhao, Effect of molecular weight on the properties of 1079 chitosan films prepared using electrostatic spraying technique, Carbohydrate polymers 212 1080 (2019) 197-205.

1081 [25] N.V. Zakharova, M.A. Simonova, S.N. Zelinskii, V.V. Annenkov, A.P. Filippov, Synthesis, 1082 molecular characteristics, and stimulus-sensitivity of graft copolymer of chitosan and poly (N, N1083 diethylacrylamide), Journal of Molecular Liquids 292 (2019) 111355.

1084 [26] G.L. Dotto, L. Pinto, General considerations about Chitosan, Materials and its applications. 1085 Bentham Science Publishers, Sharjah (2017) 3-33.

1086 [27] G. Kapadnis, A. Dey, P. Dandekar, R. Jain, Effect of degree of deacetylation on solubility of 1087 low-molecular-weight chitosan produced via enzymatic breakdown of chitosan, Polymer 1088 International 68(6) (2019) 1054-1063.

1089 [28] W. Li, G. Cai, P. Zhang, A simple and rapid Fourier transform infrared method for the 1090 determination of the degree of acetyl substitution of cellulose nanocrystals, Journal of materials 1091 science 54(10) (2019) 8047-8056.

1092 [29] U. Habiba, T.C. Joo, T.A. Siddique, A. Salleh, B.C. Ang, A.M. Afifi, Effect of degree of 1093 deacetylation of chitosan on adsorption capacity and reusability of chitosan/polyvinyl 1094 alcohol/ $/ \mathrm{TiO}_{2}$ nano composite, International journal of biological macromolecules 104 (2017) $1095 \quad 1133-1142$.

1096 [30] J.O. Gonçalves, D.A. Duarte, G.L. Dotto, L.A.A. Pinto, Use of chitosan with different 1097 deacetylation degrees for the adsorption of food dyes in a binary system, CLEAN-Soil, Air, 1098 Water 42(6) (2014) 767-774.

1099 [31] C. Zhuang, Y. Zhong, Y. Zhao, Effect of deacetylation degree on properties of Chitosan 1100 films using electrostatic spraying technique, Food control 97 (2019) 25-31.

1101 [32] A. Dodero, E. Brunengo, M. Alloisio, A. Sionkowska, S. Vicini, M. Castellano, Chitosan1102 based electrospun membranes: effects of solution viscosity, coagulant and crosslinker, 1103 Carbohydrate Polymers 235 (2020) 115976.

1104 [33] W. Thakhiew, S. Devahastin, S. Soponronnarit, Physical and mechanical properties of 1105 chitosan films as affected by drying methods and addition of antimicrobial agent, Journal of food 1106 engineering 119(1) (2013) 140-149. 
[34] E. Jakubowska, M. Gierszewska, J. Nowaczyk, E. Olewnik-Kruszkowska, Physicochemical and storage properties of chitosan-based films plasticized with deep eutectic solvent, Food Hydrocolloids 108 (2020) 106007.

1110 [35] C. López-Iglesias, J. Barros, I. Ardao, F.J. Monteiro, C. Alvarez-Lorenzo, J.L. Gómez1111 Amoza, C.A. García-González, Vancomycin-loaded chitosan aerogel particles for chronic wound 1112 applications, Carbohydrate polymers 204 (2019) 223-231.

1113 [36] M. Monier, I. Youssef, D. Abdel-Latif, Synthesis of photo-responsive chitosan-cinnamate 1114 for efficient entrapment of $\beta$-galactosidase enzyme, Reactive and Functional Polymers 124 1115 (2018) 129-138.

1116 [37] T.R. Cadaval, G.L. Dotto, L.A. Pinto, Equilibrium isotherms, thermodynamics, and kinetic 1117 studies for the adsorption of food azo dyes onto chitosan films, Chemical Engineering 1118 Communications 202(10) (2015) 1316-1323.

1119 [38] N.H. Elsayed, M. Monier, I. Youssef, Fabrication of photo-active trans-3-(4-pyridyl) acrylic 1120 acid modified chitosan, Carbohydrate polymers 172 (2017) 1-10.

1121 [39] A. Menazea, M.H. El-Newehy, B.M. Thamer, M.E. El-Naggar, Synthesis, characterization 1122 and antibacterial activity of poly (vinyl alcohol)/chitosan film embedded with the as-prepared 1123 vanadium oxide nanoparticles via laser ablation, Journal of Molecular Structure (2020) 129163.

1124 [40] S. Wei, Y.C. Ching, C.H. Chuah, Synthesis of chitosan aerogels as promising carriers for 1125 drug delivery: A review, Carbohydrate Polymers 231 (2020) 115744.

1126 [41] N.D. Aljbour, M.D. Beg, J. Gimbun, Acid Hydrolysis of Chitosan to Oligomers Using 1127 Hydrochloric Acid, Chemical Engineering \& Technology 42(9) (2019) 1741-1746.

1128 [42] C. Duan, X. Meng, J. Meng, M.I.H. Khan, L. Dai, A. Khan, X. An, J. Zhang, T. Huq, Y. Ni, 1129 Chitosan as a preservative for fruits and vegetables: a review on chemistry and antimicrobial 1130 properties, Journal of Bioresources and Bioproducts 4(1) (2019) 11-21.

1131 [43] M.M. Fouda, R. Wittke, D. Knittel, E. Schollmeyer, Use of chitosan/polyamine biopolymers 1132 based cotton as a model system to prepare antimicrobial wound dressing, International Journal of 1133 Diabetes Mellitus 1(1) (2009) 61-64.

1134 [44] N.Z. Shaban, A.M. Aboelsaad, K.R. Shoueir, S.A. Abdulmalek, D. Awad, S.Y. Shaban, H. 1135 Mansour, Chitosan-based dithiophenolato nanoparticles: Preparation, mechanistic information of 1136 DNA binding, antibacterial and cytotoxic activities, Journal of Molecular Liquids 318 (2020) $1137 \quad 114252$. 
[45] A.A. El-Bindary, E.A. Toson, K.R. Shoueir, H.A. Aljohani, M.M. Abo-Ser, Metal-organic

1139 frameworks as efficient materials for drug delivery: Synthesis, characterization, antioxidant, 1140 anticancer, antibacterial and molecular docking investigation, Applied Organometallic Chemistry $114134(2020) 5905$.

1142 [46] B.H. Beck, M. Yildirim-Aksoy, C.A. Shoemaker, S.A. Fuller, E. Peatman, Antimicrobial 1143 activity of the biopolymer chitosan against Streptococcus iniae, Journal of fish diseases 42(3) 1144 (2019) 371-377.

1145 [47] M.S.S. Abadi, E. Mirzaei, A. Bazargani, A. Gholipour, H. Heidari, N. Hadi, Antibacterial 1146 activity and mechanism of action of chitosan nanofibers against toxigenic Clostridioides 1147 (Clostridium) difficile Isolates, Ann Ig 32(1) (2020) 72-80.

1148 [48] Teaima, M. H., Abdelnaby, F. A., Fadel, M., El-Nabarawi, M. A., \& Shoueir, K. R. (2020). 1149 Synthesis of Biocompatible and Environmentally Nanofibrous Mats Loaded with Moxifloxacin 1150 as a Model Drug for Biomedical Applications. Pharmaceutics, 12(11), 1029.

1151 [49] M.H. Teaima, M.K. Elasaly, S.A. Omar, M.A. El-Nabarawi, K.R. Shoueir, Eco-friendly 1152 synthesis of functionalized chitosan-based nanoantibiotic system for potential delivery of 1153 linezolid as antimicrobial agents, Saudi Pharmaceutical Journal 28 (2020) 859-968.

1154 [50] D. Aluani, V. Tzankova, M. Kondeva-Burdina, Y. Yordanov, E. Nikolova, F. Odzhakov, A. 1155 Apostolov, T. Markova, K. Yoncheva, Evaluation of biocompatibility and antioxidant efficiency 1156 of chitosan-alginate nanoparticles loaded with quercetin, International journal of biological 1157 macromolecules 103 (2017) 771-782.

1158 [51] A.M. El-Shafei, M.M. Fouda, D. Knittel, E. Schollmeyer, Antibacterial activity of 1159 cationically modified cotton fabric with carboxymethyl chitosan, Journal of Applied Polymer 1160 Science 110(3) (2008) 1289-1296.

1161 [52] M. Ahmed, A.M. Moydeen, A. Ismail, M.E. El-Naggar, A. Menazea, M.H. El-Newehy, 1162 Polymeric blends of chitosan/poly (vinyl alcohol) containing selenium nanoparticlessynthesized 1163 by green method for wound dressing applications, Journal of Molecular Structure (2020) 1164129138.

1165 [53] R.F. Elshaarawy, G.A. Seif, M.E. El-Naggar, T.B. Mostafa, E.A. El-Sawi, In-situ and ex-situ 1166 synthesis of poly-(imidazolium vanillyl)-grafted chitosan/silver nanobiocomposites for safe 1167 antibacterial finishing of cotton fabrics, European Polymer Journal 116 (2019) 210-221. 
[54] J.A. Badano, N.V. Braber, Y. Rossi, L.D. Vergara, L. Bohl, C. Porporatto, R.D. Falcone, M. Montenegro, Physicochemical, in vitro antioxidant and cytotoxic properties of water-soluble chitosan-lactose derivatives, Carbohydrate polymers 224 (2019) 115158.

[55] F. Sami El-banna, M.E. Mahfouz, S. Leporatti, M. El-Kemary, N. AN Hanafy, Chitosan as a Natural Copolymer with Unique Properties for the Development of Hydrogels, Applied Sciences 9(11) (2019) 2193.

[56] A.M. Atta, G.A. El-Mahdy, H.A. Al-Lohedan, K.R. Shoueir, Electrochemical behavior of smart $\mathrm{N}$-isopropyl acrylamide copolymer nanogel on steel for corrosion protection in acidic solution, Int. J. Electrochem. Sci 10 (2015) 870-882.

[57] S.M. Nasef, E.E. Khozemy, E.A. Kamoun, H. El-Gendi, Gamma radiation-induced crosslinked composite membranes based on polyvinyl alcohol/chitosan/ $\mathrm{AgNO}_{3} / \mathrm{vitamin} \mathrm{E}$ for biomedical applications, International journal of biological macromolecules 137 (2019) 878-885. [58] Y.-H. Cheng, T.-H. Tsai, Y.-Y. Jhan, A.W.-h. Chiu, K.-L. Tsai, C.-S. Chien, S.-H. Chiou, C.J.-1. Liu, Thermosensitive chitosan-based hydrogel as a topical ocular drug delivery system of latanoprost for glaucoma treatment, Carbohydrate polymers 144 (2016) 390-399.

[59] L. Fan, H. Yang, J. Yang, M. Peng, J. Hu, Preparation and characterization of chitosan/gelatin/PVA hydrogel for wound dressings, Carbohydrate polymers 146 (2016) 427434.

[60] S. Naskar, S. Sharma, K. Kuotsu, Chitosan-based nanoparticles: An overview of biomedical applications and its preparation, Journal of Drug Delivery Science and Technology 49 (2019) 6681.

[61] R.R. Fouad, H.A. Aljohani, K.R. Shoueir, Biocompatible poly (vinyl alcohol) nanoparticlebased binary blends for oil spill control, Marine Pollution Bulletin 112(1-2) (2016) 46-52.

[62] M.E. El-Naggar, K. Shoueir, Recent advances in polymer/metal/metal oxide hybrid nanostructures for catalytic applications, Journal of Environmental Chemical Engineering 8 (2020) 104175.

[63] N.Z. Shaban, S.A. Yehia, K.R. Shoueir, S.R. Saleh, D. Awad, S.Y. Shaban, Design, DNA binding and kinetic studies, antibacterial and cytotoxic activities of stable dithiophenolato titanium (IV)-chitosan Nanocomposite, Journal of Molecular Liquids 287 (2019) 111002.

[64] U. Garg, S. Chauhan, U. Nagaich, N. Jain, Current advances in chitosan nanoparticles based drug delivery and targeting, Advanced pharmaceutical bulletin 9(2) (2019) 195. 
1199

1200

1201

1202

1203

1204

1205

1206

1207

1208

1209

1210

1211

1212

1213

1214

1215

1216

1217

1218

1219

1220

1221

1222

1223

1224

1225

1226

1227

1228

1229

[65] Y. Ohya, M. Shiratani, H. Kobayashi, T. Ouchi, Release behavior of 5-fluorouracil from chitosan-gel nanospheres immobilizing 5-fluorouracil coated with polysaccharides and their cell specific cytotoxicity, Journal of Macromolecular Science-Pure and Applied Chemistry 31(5) (1994) 629-642.

[66] D. Patiño-Ruiz, L. Marrugo, N. Reyes, M. Acevedo-Morantes, A. Herrera, Ionotropic Gelation Synthesis of Chitosan-Alginate Nanodisks for Delivery System and In Vitro Assessment of Prostate Cancer Cytotoxicity, International Journal of Polymer Science 2020 (2020).

[67] F.G. de Carvalho, T.C. Magalhães, N.M. Teixeira, B.L.C. Gondim, H.L. Carlo, R.L. Dos Santos, A.R. de Oliveira, Â.M.L. Denadai, Synthesis and characterization of TPP/chitosan nanoparticles: Colloidal mechanism of reaction and antifungal effect on C. albicans biofilm formation, Materials Science and Engineering: C 104 (2019) 109885.

[68] Y. Nemati, P. Zahedi, M. Baghdadi, S. Ramezani, Microfluidics combined with ionic gelation method for production of nanoparticles based on thiol-functionalized chitosan to adsorb $\mathrm{Hg}$ (II) from aqueous solutions, Journal of environmental management 238 (2019) 166-177.

[69] K. Divya, M. Jisha, Chitosan nanoparticles preparation and applications, Environmental chemistry letters 16(1) (2018) 101-112.

[70] A.C. Pessoa, C.C. Sipoli, G. Lucimara, Effects of diffusion and mixing pattern on microfluidic-assisted synthesis of chitosan/ATP nanoparticles, Lab on a Chip 17(13) (2017) 2281-2293.

[71] L. Bugnicourt, C. Ladavière, Interests of chitosan nanoparticles ionically cross-linked with tripolyphosphate for biomedical applications, Progress in polymer science 60 (2016) 1-17.

[72] X. Wang, J. Shi, Z. Li, S. Zhang, H. Wu, Z. Jiang, C. Yang, C. Tian, Facile one-pot preparation of chitosan/calcium pyrophosphate hybrid microflowers, ACS applied materials \& interfaces 6(16) (2014) 14522-14532.

[73] D. Komoto, T. Furuike, H. Tamura, Preparation of polyelectrolyte complex gel of sodium alginate with chitosan using basic solution of chitosan, International journal of biological macromolecules 126 (2019) 54-59.

[74] M. Boudoukhani, M.M. Yahoum, S. Lefnaoui, N. Moulai-Mostefa, M. Banhobre, Synthesis, characterization and evaluation of deacetylated xanthan derivatives as new excipients in the formulation of chitosan-based polyelectrolytes for the sustained release of tramadol, Saudi Pharmaceutical Journal 27(8) (2019) 1127-1137. 
1230 [75] K. Livanovich, T. Shutava, Influence of chitosan/dextran sulfate layer-by-layer shell on 1231 colloidal properties of silver nanoparticles, International Journal of Nanoscience 18 (2019) 12321940077.

1233 [76] M.K. Haidar, G.M. Demirbolat, S.S. Timur, R.N. Gürsoy, E. Nemutlu, K. Ulubayram, L. 1234 Öner, H. Eroğlu, Atorvastatin-loaded nanosprayed chitosan nanoparticles for peripheral nerve 1235 injury, Bioinspired, Biomimetic and Nanobiomaterials 9 (2020) 74-84.

1236 [77] T.A. Ahmed, B.M. Aljaeid, Preparation, characterization, and potential application of 1237 chitosan, chitosan derivatives, and chitosan metal nanoparticles in pharmaceutical drug delivery, 1238 Drug design, development and therapy 10 (2016) 483.

1239 [78] K. Lech, Quality and Safety Aspects of Spray-Dried Foods, Handbook on Spray Drying 1240 Applications for Food Industries (2019) 307.

1241 [79] J. Lucas, M. Ralaivao, B.N. Estevinho, F. Rocha, A new approach for the 1242 microencapsulation of curcumin by a spray drying method, in order to value food products, 1243 Powder Technology 362 (2020) 428-435.

1244 [80] C. Li, C. Crua, K. Vogiatzaki, Effect of the scale resolution on the two phase coupling 1245 characteristics of high speed evaporating sprays using LES/Eulerian-Lagrangian methodologies, 1246 International Journal of Multiphase Flow 120 (2019) 103060.

1247 [81] U. Luesakul, S. Puthong, K. Sansanaphongpricha, N. Muangsin, Quaternized chitosan1248 coated nanoemulsions: A novel platform for improving the stability, anti-inflammatory, anti1249 cancer and transdermal properties of plai extract, Carbohydrate Polymers 230 (2020) 115625.

1250 [82] S. Chaudhary, S. Kumar, V. Kumar, R. Sharma, Chitosan nanoemulsions as advanced edible 1251 coatings for fruits and vegetables: Composition, fabrication and developments in last decade, 1252 International Journal of Biological Macromolecules 152 (2020) 154-170.

1253 [83] L. Liu, H. Yang, Y. Lou, J.-Y. Wu, J. Miao, X.-Y. Lu, J.-Q. Gao, Enhancement of oral 1254 bioavailability of salmon calcitonin through chitosan-modified, dual drug-loaded nanoparticles, 1255 International journal of pharmaceutics 557 (2019) 170-177.

1256 [84] A. Yu, H. Shi, H. Liu, Z. Bao, M. Dai, D. Lin, D. Lin, X. Xu, X. Li, Y. Wang, 1257 Mucoadhesive dexamethasone-glycol chitosan nanoparticles for ophthalmic drug delivery, 1258 International Journal of Pharmaceutics 575 (2020) 118943. 
1259 [85] H. Zhang, X. Li, H. Kang, Chitosan coatings incorporated with free or nano-encapsulated

1260 Paulownia Tomentosa essential oil to improve shelf-life of ready-to-cook pork chops, LWT 116 1261 (2019) 108580.

1262 [86] T. Xu, Y. Ma, J. Huang, H. Lai, D. Yuan, X. Tang, L. Yang, Self-organized thermo1263 responsive poly (lactic-co-glycolic acid)-graft-pullulan nanoparticles for synergistic thermo1264 chemotherapy of tumor, Carbohydrate Polymers (2020) 116104.

1265 [87] H. Roy, B.S. Nayak, S. Nandi, Chitosan Anchored Nanoparticles in Current Drug 1266 Development Utilizing Computer-Aided Pharmacokinetic Modeling: Case Studies for Target 1267 Specific Cancer Treatment and Future Prospective, Current Pharmaceutical Design 26 (2020) 1268 1666-1675.

1269 [88] M.M. Khan, A. Madni, V. Torchilin, N. Filipczak, J. Pan, N. Tahir, H. Shah, Lipid-chitosan 1270 hybrid nanoparticles for controlled delivery of cisplatin, Drug Delivery 26(1) (2019) 765-772.

1271 [89] Z. Du, J. Liu, T. Zhang, Y. Yu, Y. Zhang, J. Zhai, H. Huang, S. Wei, L. Ding, B. Liu, A 1272 study on the preparation of chitosan-tripolyphosphate nanoparticles and its entrapment 1273 mechanism for egg white derived peptides, Food chemistry 286 (2019) 530-536.

1274 [90] M. Ji, X. Sun, X. Guo, W. Zhu, J. Wu, L. Chen, J. Wang, M. Chen, C. Cheng, Q. Zhang, 1275 Green synthesis, characterization and in vitro release of cinnamaldehyde/sodium 1276 alginate/chitosan nanoparticles, Food hydrocolloids 90 (2019) 515-522.

1277 [91] S. Omidi, A. Kakanejadifard, Modification of chitosan and chitosan nanoparticle by long 1278 chain pyridinium compounds: Synthesis, characterization, antibacterial, and antioxidant 1279 activities, Carbohydrate polymers 208 (2019) 477-485.

1280 [92] Y. Guo, M. Dai, Z. Zhu, Y. Chen, H. He, T. Qin, Chitosan modified Cu2O nanoparticles 1281 with high catalytic activity for p-nitrophenol reduction, Applied Surface Science 480 (2019) 6011282610.

1283 [93] M. Vakili, S. Deng, G. Cagnetta, W. Wang, P. Meng, D. Liu, G. Yu, Regeneration of 1284 chitosan-based adsorbents used in heavy metal adsorption: A review, Separation and Purification 1285 Technology 224 (2019) 373-387.

1286 [94] M. Vakili, S. Deng, D. Liu, T. Li, G. Yu, Preparation of aminated cross-linked chitosan 1287 beads for efficient adsorption of hexavalent chromium, International journal of biological 1288 macromolecules 139 (2019) 352-360. 

Ternary adsorption of cobalt, nickel and methylene blue on a modified chitin: Phenomenological modeling and physical interpretation of the adsorption mechanism, International Journal of

1292 Biological Macromolecules 158 (2020) 595-604.

1293 [96] K.R. Shoueir, Green microwave synthesis of functionalized chitosan with robust adsorption 1294 capacities for $\mathrm{Cr}$ (VI) and/or RHB in complex aqueous solutions, Environmental Science and 1295 Pollution Research 27 (2020) 33020-33031.

1296 [97] H. Merzendorfer, E. Cohen, Chitin/Chitosan: Versatile Ecological, Industrial, and 1297 Biomedical Applications, Extracellular Sugar-Based Biopolymers Matrices (2019) 541-624.

1298 [98] S.R. Nadendla, T.S. Rani, P.R. Vaikuntapu, R.R. Maddu, A.R. Podile, HarpinPss 1299 encapsulation in chitosan nanoparticles for improved bioavailability and disease resistance in 1300 tomato, Carbohydrate polymers 199 (2018) 11-19.

1301 [99] C.R. de Oliveira, L.F. Fraceto, G.M. Rizzi, R.F. Salla, F.C. Abdalla, M.J. Costa, E.C.M. 1302 Silva-Zacarin, Hepatic effects of the clomazone herbicide in both its free form and associated 1303 with chitosan-alginate nanoparticles in bullfrog tadpoles, Chemosphere 149 (2016) 304-313. [100] A.K. Dhakad, M. Ikram, S. Sharma, S. Khan, V.V. Pandey, A. Singh, Biological, 1305 nutritional, and therapeutic significance of Moringa oleifera Lam, Phytotherapy Research 33(11) 1306 (2019) 2870-2903. of gelatin films containing tea polyphenol-loaded chitosan nanoparticles generated by electrospray, Materials \& Design 185 (2020) 108277. polysaccharides-based nanoparticles to improve sustained antimicrobial activity of nisin, 1312 Carbohydrate polymers 225 (2019) 115251.

1313 [103] K. Hajiasgharzadeh, M.H. Somi, D. Shanehbandi, A. Mokhtarzadeh, B. Baradaran, Small 1314 interfering RNA-mediated gene suppression as a therapeutic intervention in hepatocellular 1315 carcinoma, Journal of Cellular Physiology 234(4) (2019) 3263-3276.

1316 [104] N. Laroui, M. Coste, L. Lichon, Y. Bessin, M. Gary-Bobo, G. Pratviel, C. Bonduelle, N. 1317 Bettache, S. Ulrich, Combination of photodynamic therapy and gene silencing achieved through 1318 the hierarchical self-assembly of porphyrin-siRNA complexes, International journal of 1319 pharmaceutics 569 (2019) 118585. 
1320 [105] W. Zhang, W. Xu, Y. Lan, X. He, K. Liu, Y. Liang, Antitumor effect of hyaluronic-acid1321 modified chitosan nanoparticles loaded with siRNA for targeted therapy for non-small cell lung 1322 cancer, International Journal of Nanomedicine 14 (2019) 5287.

1323 [106] F. Chaharband, N. Daftarian, M.R. Kanavi, R. Varshochian, M. Hajiramezanali, P. 1324 Norouzi, E. Arefian, F. Atyabi, R. Dinarvand, Trimethyl chitosan-hyaluronic acid nano1325 polyplexes for intravitreal VEGFR-2 siRNA delivery: Formulation and in-vivo efficacy 1326 evaluation, Nanomedicine: Nanotechnology, Biology and Medicine 26 (2020) 102181.

1327 [107] K. Yoncheva, M. Merino, A. Shenol, N.T. Daskalov, P.S. Petkov, G.N. Vayssilov, M.J. 1328 Garrido, Optimization and in-vitro/in-vivo evaluation of doxorubicin-loaded chitosan-alginate 1329 nanoparticles using a melanoma mouse model, International journal of pharmaceutics 556 (2019) $1330 \quad 1-8$.

1331 [108] K.X. Tan, S. Pan, J. Jeevanandam, M.K. Danquah, Cardiovascular therapies utilizing 1332 targeted delivery of nanomedicines and aptamers, International Journal of Pharmaceutics 558 1333 (2019) 413-425.

1334 [109] J. Bruniaux, E. Allard-Vannier, N. Aubrey, Z. Lakhrif, S.B. Djemaa, S. Eljack, H. 1335 Marchais, K. Hervé-Aubert, I. Chourpa, S. David, Magnetic nanocarriers for the specific delivery 1336 of siRNA: Contribution of breast cancer cells active targeting for down-regulation efficiency, 1337 International journal of pharmaceutics 569 (2019) 118572.

1338 [110] U.H. Langen, S. Ayloo, C. Gu, Development and cell biology of the blood-brain barrier, 1339 Annual review of cell and developmental biology 35 (2019) 591-613.

1340 [111] F. Rinaldi, L. Seguella, S. Gigli, P. Hanieh, E. Del Favero, L. Cantù, M. Pesce, G. Sarnelli, 1341 C. Marianecci, G. Esposito, inPentasomes: An innovative nose-to-brain pentamidine delivery 1342 blunts MPTP parkinsonism in mice, Journal of Controlled Release 294 (2019) 17-26.

1343 [112] J.S. Ling Tan, C.J. Roberts, N. Billa, Mucoadhesive chitosan-coated nanostructured lipid 1344 carriers for oral delivery of amphotericin B, Pharmaceutical development and technology 24(4) 1345 (2019) 504-512.

1346 [113] S. Rehman, B. Nabi, A. Zafar, S. Baboota, J. Ali, Intranasal delivery of mucoadhesive 1347 nanocarriers: a viable option for Parkinson's disease treatment?, Expert Opinion on Drug 1348 Delivery 16(12) (2019) 1355-1366. 
1349 [114] I. Schlachet, A. Sosnik, Mixed Mucoadhesive Amphiphilic Polymeric Nanoparticles Cross 1350 a Model of Nasal Septum Epithelium in Vitro, ACS applied materials \& interfaces 11(24) (2019) $1351 \quad 21360-21371$.

1352 [115] V.S. Lima, A.T.B. Guimarães, A.P. da Costa Araújo, F.N. Estrela, I.C. da Silva, N.F.S. de 1353 Melo, L.F. Fraceto, G. Malafaia, Depression, anxiety-like behavior, and memory impairment in 1354 mice exposed to chitosan-coated zein nanoparticles, Environmental Science and Pollution 1355 Research 26(11) (2019) 10641-10650.

1356 [116] Y. Zhao, D. Li, Z. Zhu, Y. Sun, Improved neuroprotective effects of gallic acid-loaded 1357 chitosan nanoparticles against ischemic stroke, Rejuvenation Research (2019).

1358 [117] G. Arumugam, R. Rajendran, Callophycin A loaded chitosan and spicules based 1359 nanocomposites as an alternative strategy to overcome vaginal candidiasis, International Journal 1360 of Biological Macromolecules 161 (2020) 656-665.

1361 [118] U. Arif, S. Haider, A. Haider, N. Khan, A.A. Alghyamah, N. Jamila, M.I. Khan, W.A. 1362 Almasry, I.-K. Kang, Biocompatible Polymers and their Potential Biomedical Applications: A 1363 Review, Current Pharmaceutical Design 25(34) (2019) 3608-3619.

1364 [119] T.-W. Huang, Y.-C. Ho, T.-N. Tsai, C.-L. Tseng, C. Lin, F.-L. Mi, Enhancement of the 1365 permeability and activities of epigallocatechin gallate by quaternary ammonium 1366 chitosan/fucoidan nanoparticles, Carbohydrate Polymers 242 (2020) 116312.

1367 [120] A. Andleeb, M. Yar, Application of Electrospun Materials in Industrial Applications, 1368 Electrospun Materials and Their Allied Applications (2020) 215-242.

1369 [121] C. Wang, J. Wang, L. Zeng, Z. Qiao, X. Liu, H. Liu, J. Zhang, J. Ding, Fabrication of 1370 electrospun polymer nanofibers with diverse morphologies, Molecules 24(5) (2019) 834.

1371 [122] M.F. Abdelbar, H.S. El-Sheshtawy, K.R. Shoueir, I. El-Mehasseb, E.-Z.M. Ebeid, M. El1372 Kemary, Halogen bond triggered aggregation induced emission in an iodinated cyanine dye for 1373 ultra sensitive detection of Ag nanoparticles in tap water and agricultural wastewater, RSC 1374 advances 8(43) (2018) 24617-24626.

1375 [123] K.R. Shoueir, A.M. Atta, A.A. Sarhan, M.A. Akl, Synthesis of monodisperse core shell 1376 PVA@ P (AMPS-co-NIPAm) nanogels structured for pre-concentration of Fe (III) ions, 1377 Environmental technology 38(8) (2017) 967-978. 
1378 [124] H.S. El-Sheshtawy, K.R. Shouir, M. El-Kemary, Activated $\mathrm{H}_{2} \mathrm{O}_{2}$ on $\mathrm{Ag} / \mathrm{SiO}_{2}-\mathrm{SrWO}_{4}$ 1379 surface for enhanced dark and visible-light removal of methylene blue and p-nitrophenol, Journal 1380 of Alloys and Compounds 842 (2020) 155848.

1381 [125] K. Shoueir, A.R. Wassel, M. Ahmed, M.E. El-Naggar, Encapsulation of extremely stable 1382 polyaniline onto Bio-MOF: Photo-activated antibacterial and depletion of ciprofloxacin from 1383 aqueous solutions, Journal of Photochemistry and Photobiology A: Chemistry 400 (2020) 1384112703.

1385 [126] K.R. Shoueir, A. Sarhan, A.M. Atta, M.A. Akl, Adsorption studies of $\mathrm{Cu}^{2+}$ onto poly (vinyl 1386 alcohol)/poly (acrylamide-co-N-isopropylacrylamide) core-shell nanogels synthesized through 1387 surfactant-free emulsion polymerization, Separation Science and Technology 51(10) (2016) $1388 \quad 1605-1617$.

1389 [127] S. Guo, L. He, R. Yang, B. Chen, X. Xie, B. Jiang, T. Weidong, Y. Ding, Enhanced effects 1390 of electrospun collagen-chitosan nanofiber membranes on guided bone regeneration, Journal of 1391 Biomaterials Science, Polymer Edition 31(2) (2020) 155-168.

1392 [128] M. Mohraz, F. Golbabaei, I. Yu, M. Mansournia, A. Zadeh, S. Dehghan, Preparation and 1393 optimization of multifunctional electrospun polyurethane/chitosan nanofibers for air pollution 1394 control applications, International Journal of Environmental Science and Technology 16(2) 1395 (2019) 681-694.

1396 [129] M. Séon-Lutz, A.-C. Couffin, S. Vignoud, G. Schlatter, A. Hébraud, Electrospinning in 1397 water and in situ crosslinking of hyaluronic acid/cyclodextrin nanofibers: Towards wound 1398 dressing with controlled drug release, Carbohydrate polymers 207 (2019) 276-287.

1399 [130] M.P. Figueiredo, G. Layrac, A. Hébraud, L. Limousy, J. Brendle, G. Schlatter, V.R. 1400 Constantino, Design of 3D multi-layered electrospun membranes embedding iron-based layered 1401 double hydroxide for drug storage and control of sustained release, European Polymer Journal $1402131(2020) 109675$.

1403 [131] F. Wang, Z. Sun, J. Yin, L. Xu, Preparation, characterization and properties of porous $1404 \mathrm{pla} / \mathrm{peg} / \mathrm{curcumin}$ composite nanofibers for antibacterial application, Nanomaterials 9(4) (2019) 1405508.

1406 [132] Y. Liu, C. Wang, Advanced Nanofibrous Materials Manufacture Technology Based on 1407 Electrospinning, CRC Press2019. 
1408 [133] C.-H. He, Y. Shen, F.-Y. Ji, J.-H. He, Taylor series solution for fractal Bratu-type equation 1409 arising in electrospinning process, Fract 28(1) (2020) 2050011-2193.

1410 [134] R. Ramakrishnan, J. Gimbun, P. Ramakrishnan, B. Ranganathan, S.M. Reddy, G. 1411 Shanmugam, Effect of solution properties and operating parameters on needleless electrospinning 1412 of poly (ethylene oxide) nanofibers loaded with bovine serum albumin, Current Drug Delivery $141316(10)(2019) 913-922$.

1414 [135] M. Liang, A. Hébraud, G. Schlatter, Modeling and on-line measurement of the surface 1415 potential of electrospun membranes for the control of the fiber diameter and the pore size, 1416 Polymer $200(2020) 122576$.

1417 [136] A. Panahi, A.R. Pishevar, M.R. Tavakoli, Numerical Simulation of Jet Mode in 1418 Electrospraying of Newtonian and Viscoelastic Fluids, International Journal of Multiphase Flow $1419129(2020) 103302$.

1420 [137] C.G. Reyes, J.P. Lagerwall, Disruption of electrospinning due to water condensation into 1421 the Taylor cone, ACS Applied Materials \& Interfaces (2020) DOI: 10.1021/acsami.0c03338.

1422 [138] A. Iregui, L. Irusta, L. Martin, A. González, Analysis of the process parameters for 1423 obtaining a stable electrospun process in different composition epoxy/poly $\varepsilon$-Caprolactone blends 1424 with shape memory properties, Polymers 11(3) (2019) 475.

1425 [139] M. Allais, D. Mailley, P. Hébraud, D. Ihiawakrim, V. Ball, F. Meyer, A. Hébraud, G. 1426 Schlatter, Polymer-free electrospinning of tannic acid and cross-linking in water for hybrid 1427 supramolecular nanofibres, Nanoscale 10(19) (2018) 9164-9173.

1428 [140] L. Chen, H. Jiang, Y. Li, B.L. Zimba, X. Yu, C. Chen, G. Xiong, Q. Wu, Influences on 1429 mechanical properties of chitosan nanofibrous membranes induced by incorporating graphene 1430 oxide nanosheets, Materials Research Express 6(7) (2019) 075404.

1431 [141] S. Afshar, S. Rashedi, H. Nazockdast, M. Ghazalian, Preparation and characterization of 1432 electrospun poly (lactic acid)-chitosan core-shell nanofibers with a new solvent system, 1433 International journal of biological macromolecules 138 (2019) 1130-1137.

1434 [142] S. Ebrahimi, M. Fathi, M. Kadivar, Production and characterization of chitosan-gelatin 1435 nanofibers by nozzle-less electrospinning and their application to enhance edible film's 1436 properties, Food Packaging and Shelf Life 22 (2019) 100387. 
1437 [143] M. Wang, T. Hai, Z. Feng, D.-G. Yu, Y. Yang, S. Annie Bligh, The relationships between 1438 the working fluids, process characteristics and products from the modified coaxial 1439 electrospinning of zein, Polymers 11(8) (2019) 1287.

1440 [144] M. Zhang, X. Huang, H. Xin, D. Li, Y. Zhao, L. Shi, Y. Lin, J. Yu, Z. Yu, C. Zhu, Coaxial 1441 electrospinning synthesis hollow Mo2C@ C core-shell nanofibers for high-performance and 1442 long-term lithium-ion batteries, Applied Surface Science 473 (2019) 352-358.

1443 [145] M. Shamsipour, A.M. Mansouri, P. Moradipour, Temozolomide conjugated carbon 1444 quantum dots embedded in core/shell nanofibers prepared by coaxial electrospinning as an 1445 implantable delivery system for cell imaging and sustained drug release, AAPS PharmSciTech 1446 20(7) (2019) 259.

1447 [146] R. Rasouli, A. Barhoum, M. Bechelany, A. Dufresne, Nanofibers for biomedical and 1448 healthcare applications, Macromolecular bioscience 19(2) (2019) 1800256.

1449 [147] Z.A. Al-Ahmed, N.S. Al-Radadi, M. Ahmed, K. Shoueir, M. Elkemary, Dye removal, 1450 antibacterial properties, and morphological behavior of hydroxyapatite doped with $\mathrm{Pd}$ ions, 1451 Arabian Journal of Chemistry (2020).

1452 [148] K.R. Shoueir, M.A. Akl, A.A. Sarhan, A.M. Atta, New core@ shell nanogel based 21453 acrylamido-2-methyl-1-propane sulfonic acid for preconcentration of $\mathrm{Pb}$ (II) from various water 1454 samples, Applied Water Science 7(7) (2017) 3729-3740.

1455 [149] A. Celebioglu, F. Topuz, Z.I. Yildiz, T. Uyar, One-step green synthesis of antibacterial 1456 silver nanoparticles embedded in electrospun cyclodextrin nanofibers, Carbohydrate polymers 1457207 (2019) 471-479.

1458 [150] F. Tao, Y. Cheng, X. Shi, H. Zheng, Y. Du, W. Xiang, H. Deng, Applications of chitin and 1459 chitosan nanofibers in bone regenerative engineering, Carbohydrate Polymers 230 (2020) 1460115658.

1461 [151] J.-P. Fan, J.-J. Luo, X.-H. Zhang, B. Zhen, C.-Y. Dong, Y.-C. Li, J. Shen, Y.-T. Cheng, H.1462 P. Chen, A novel electrospun $\beta$-CD/CS/PVA nanofiber membrane for simultaneous and rapid 1463 removal of organic micropollutants and heavy metal ions from water, Chemical Engineering 1464 Journal 378 (2019) 122232.

1465 [152] N.S. Surgutskaia, A. Di Martino, J. Zednik, K. Ozaltin, L. Lovecká, E.D. Bergerová, D. 1466 Kimmer, J. Svoboda, V. Sedlarik, Efficient $\mathrm{Cu} 2+, \mathrm{Pb} 2+$ and $\mathrm{Ni} 2+$ ion removal from wastewater 
1467 using electrospun DTPA-modified chitosan/polyethylene oxide nanofibers, Separation and 1468 Purification Technology 247 (2020) 116914.

1469 [153] K. Shoueir, S. Kandil, H. El-hosainy, M. El-Kemary, Tailoring the surface reactivity of 1470 plasmonic $\mathrm{Au} @ \mathrm{TiO}_{2}$ photocatalyst bio-based chitosan fiber towards cleaner of harmful water 1471 pollutants under visible-light irradiation, Journal of Cleaner Production 230 (2019) 383-393.

1472 [154] A.S. Shabunin, V.E. Yudin, I.P. Dobrovolskaya, E.V. Zinovyev, V. Zubov, E.M. 1473 Ivan'kova, P. Morganti, Composite wound dressing based on chitin/chitosan nanofibers: 1474 processing and biomedical applications, Cosmetics 6(1) (2019) 16.

1475 [155] S. Fahimirad, F. Ajalloueian, Naturally-derived electrospun wound dressings for target 1476 delivery of bio-active agents, International Journal of Pharmaceutics 566 (2019) 307-328.

1477 [156] L. Chen, H. Pan, C. Zhuang, M. Peng, L. Zhang, Joint wound healing using polymeric 1478 dressing of chitosan/strontium-doped titanium dioxide with high antibacterial activity, Materials 1479 Letters $268(2020) 127555$.

1480 [157] A.J. Hassiba, M.E. El Zowalaty, T.J. Webster, A.M. Abdullah, G.K. Nasrallah, K.A. 1481 Khalil, A.S. Luyt, A.A. Elzatahry, Synthesis, characterization, and antimicrobial properties of 1482 novel double layer nanocomposite electrospun fibers for wound dressing applications, 1483 International Journal of Nanomedicine 12 (2017) 2205.

1484 [158] A. Naeimi, M. Payandeh, A.R. Ghara, F.E. Ghadi, In vivo evaluation of the wound healing 1485 properties of bio-nanofiber chitosan/polyvinyl alcohol incorporating honey and Nepeta 1486 dschuparensis, Carbohydrate Polymers 240 (2020) 116315.

1487 [159] F. Hafezi, N. Scoutaris, D. Douroumis, J. Boateng, 3D printed chitosan dressing 1488 crosslinked with genipin for potential healing of chronic wounds, International journal of 1489 pharmaceutics 560 (2019) 406-415.

1490 [160] N.M. Ergul, S. Unal, I. Kartal, C. Kalkandelen, N. Ekren, O. Kilic, L. Chi-Chang, O. 1491 Gunduz, 3D printing of chitosan/ poly(vinyl alcohol) hydrogel containing synthesized 1492 hydroxyapatite scaffolds for hard-tissue engineering, Polymer Testing 79 (2019) 106006.

1493 [161] M. Zhu, X. Liu, L. Tan, Z. Cui, Y. Liang, Z. Li, K.W. Kwok Yeung, S. Wu, Photo1494 responsive chitosan/Ag/MoS2 for rapid bacteria-killing, Journal of hazardous materials 383 $1495 \quad$ (2020) 121122. 
[162] A.A. Menazea, M.K. Ahmed, Wound healing activity of Chitosan/Polyvinyl Alcohol

1497 embedded by gold nanoparticles prepared by nanosecond laser ablation, Journal of Molecular 1498 Structure 1217 (2020) 128401.

1499 [163] S. Nemati, S.-j. Kim, Y.M. Shin, H. Shin, Current progress in application of polymeric 1500 nanofibers to tissue engineering, Nano convergence 6(1) (2019) 1-16.

1501 [164] A. Aidun, A. Safaei Firoozabady, M. Moharrami, A. Ahmadi, N. Haghighipour, S. 1502 Bonakdar, S. Faghihi, Graphene oxide incorporated polycaprolactone/chitosan/collagen 1503 electrospun scaffold: Enhanced osteogenic properties for bone tissue engineering, Artificial 1504 organs 43(10) (2019) E264-E281.

1505 [165] P. Das, J.-C. Remigy, J.-F. Lahitte, A.D. van der Meer, B. Garmy-Susini, C. Coetsier, S. 1506 Desclaux, P. Bacchin, Development of double porous poly ( $\varepsilon$-caprolactone)/chitosan polymer as 1507 tissue engineering scaffold, Materials Science and Engineering: C 107 (2020) 110257.

1508 [166] N.S. Sambudi, M.G. Kim, S.B. Park, The formation of web-like connection among 1509 electrospun chitosan/PVA fiber network by the reinforcement of ellipsoidal calcium carbonate, 1510 Materials Science and Engineering: C 60 (2016) 518-525.

1511 [167] M. Monier, D. Abdel-Latif, Fabrication of Au (III) ion-imprinted polymer based on thiol1512 modified chitosan, International journal of biological macromolecules 105 (2017) 777-787.

1513 [168] S. Abid, T. Hussain, A. Nazir, A. Zahir, N. Khenoussi, A novel double-layered polymeric 1514 nanofiber-based dressing with controlled drug delivery for pain management in burn wounds, 1515 Polymer Bulletin 76(12) (2019) 6387-6411.

1516 [169] N.B. Varukattu, R. Vivek, C. Rejeeth, R. Thangam, T. Ponraj, A. Sharma, S. Kannan, 1517 Nanostructured pH-responsive biocompatible chitosan coated copper oxide nanoparticles: A 1518 polymeric smart intracellular delivery system for doxorubicin in breast cancer cells, Arabian 1519 Journal of Chemistry 13(1) (2020) 2276-2286.

1520 [170] J.T. Duskey, C. Baraldi, M.C. Gamberini, I. Ottonelli, F. Da Ros, G. Tosi, F. Forni, M.A. 1521 Vandelli, B. Ruozi, Investigating Novel Syntheses of a Series of Unique Hybrid PLGA-Chitosan 1522 Polymers for Potential Therapeutic Delivery Applications, Polymers 12(4) (2020) 823.

1523 [171] E. Yan, M. Cao, Y. Wang, X. Hao, S. Pei, J. Gao, Y. Wang, Z. Zhang, D. Zhang, Gold 1524 nanorods contained polyvinyl alcohol/chitosan nanofiber matrix for cell imaging and drug 1525 delivery, Materials Science and Engineering: C 58 (2016) 1090-1097. 
1526 [172] J. Kozlowska, N. Stachowiak, A. Sionkowska, Preparation and characterization of 1527 collagen/chitosan poly (ethylene glycol)/nanohydroxyapatite composite scaffolds, Polymers for 1528 Advanced Technologies 30(3) (2019) 799-803.

1529 [173] T. Ang, F. Sultana, D. Hutmacher, Y.S. Wong, J. Fuh, X. Mo, H.T. Loh, E. Burdet, S.-H. 1530 Teoh, Fabrication of 3D chitosan-hydroxyapatite scaffolds using a robotic dispensing system, 1531 Materials science and engineering: C 20(1-2) (2002) 35-42.

1532 [174] A. Khalyfa, S. Vogt, J. Weisser, G. Grimm, A. Rechtenbach, W. Meyer, M. Schnabelrauch, 1533 Development of a new calcium phosphate powder-binder system for the 3D printing of patient 1534 specific implants, Journal of Materials Science: Materials in Medicine 18(5) (2007) 909-916.

1535 [175] F. Pahlevanzadeh, R. Emadi, A. Valiani, M. Kharaziha, S.A. Poursamar, H.R. Bakhsheshi1536 Rad, A.F. Ismail, S. RamaKrishna, F. Berto, Three-Dimensional Printing Constructs Based on the 1537 Chitosan for Tissue Regeneration: State of the Art, Developing Directions and Prospect Trends, 1538 Materials 13(11) (2020) 2663.

1539 [176] V.B. Morris, S. Nimbalkar, M. Younesi, P. McClellan, O. Akkus, Mechanical properties, 1540 cytocompatibility and manufacturability of chitosan: PEGDA hybrid-gel scaffolds by 1541 stereolithography, Annals of biomedical engineering 45(1) (2017) 286-296.

1542 [177] K. Ye, R. Felimban, K. Traianedes, S.E. Moulton, G.G. Wallace, J. Chung, A. Quigley, 1543 P.F. Choong, D.E. Myers, Chondrogenesis of infrapatellar fat pad derived adipose stem cells in 1544 3D printed chitosan scaffold, PLoS One 9(6) (2014) e99410.

1545 [178] S. Reed, G. Lau, B. Delattre, D.D. Lopez, A.P. Tomsia, B.M. Wu, Macro-and micro1546 designed chitosan-alginate scaffold architecture by three-dimensional printing and directional 1547 freezing, Biofabrication 8(1) (2016) 015003.

1548 [179] J. Huang, H. Fu, Z. Wang, Q. Meng, S. Liu, H. Wang, X. Zheng, J. Dai, Z. Zhang, BMSCs1549 laden gelatin/sodium alginate/carboxymethyl chitosan hydrogel for 3D bioprinting, Rsc 1550 Advances 6(110) (2016) 108423-108430.

1551 [180] W.E. Müller, E. Tolba, H.C. Schröder, M. Neufurth, S. Wang, T. Link, B. Al-Nawas, X. 1552 Wang, A new printable and durable N, O-carboxymethyl chitosan-Ca ${ }^{2+}-$ polyphosphate complex 1553 with morphogenetic activity, Journal of Materials Chemistry B 3(8) (2015) 1722-1730.

1554 [181] Y. Zhang, Y. Yu, I.T. Ozbolat, Direct bioprinting of vessel-like tubular microfluidic 1555 channels, Journal of nanotechnology in engineering and medicine 4(2) (2013) 020902. 
1556 [182] T. Zhu, J. Jiang, J. Zhao, S. Chen, X. Yan, Regulating preparation of functional alginate1557 chitosan three-dimensional scaffold for skin tissue engineering, International Journal of 1558 Nanomedicine 14 (2019) 8891.

1559 [183] S.F. Mansour, S.I. El-dek, S.V. Dorozhkin, M.K. Ahmed, Physico-mechanical properties of $1560 \mathrm{Mg}$ and $\mathrm{Ag}$ doped hydroxyapatite/chitosan biocomposites, New Journal of Chemistry 41(22) 1561 (2017) 13773-13783.

1562 [184] J. Liu, L. Sun, W. Xu, Q. Wang, S. Yu, J. Sun, Current advances and future perspectives of 1563 3D printing natural-derived biopolymers, Carbohydrate polymers 207 (2019) 297-316.

1564 [185] A. Ghilan, A.P. Chiriac, L.E. Nita, A.G. Rusu, I. Neamtu, V.M. Chiriac, Trends in 3D 1565 Printing Processes for Biomedical Field: Opportunities and Challenges, Journal of Polymers and 1566 the Environment 28 (2020) 1345-1367.

1567 [186] D.J. Choi, Y. Kho, S.J. Park, Y.-J. Kim, S. Chung, C.-H. Kim, Effect of cross-linking on 1568 the dimensional stability and biocompatibility of a tailored 3D-bioprinted gelatin scaffold, 1569 International journal of biological macromolecules 135 (2019) 659-667.

1570 [187] J. Gopinathan, T.N. Hao, E. Cha, C. Lee, D. Das, I. Noh, 3D printable and injectable 1571 lactoferrin-loaded carboxymethyl cellulose-glycol chitosan hydrogels for tissue engineering 1572 applications, Materials Science and Engineering: C 113 (2020) 111008.

1573 [188] T. Jiang, J.G. Munguia-Lopez, S. Flores-Torres, J. Kort-Mascort, J.M. Kinsella, Extrusion 1574 bioprinting of soft materials: An emerging technique for biological model fabrication, Applied 1575 Physics Reviews 6(1) (2019) 011310.

1576 [189] C.R. Almeida, T. Serra, M.I. Oliveira, J.A. Planell, M.A. Barbosa, M. Navarro, Impact of 1577 3-D printed PLA-and chitosan-based scaffolds on human monocyte/macrophage responses: 1578 unraveling the effect of 3-D structures on inflammation, Acta biomaterialia 10(2) (2014) 6131579622.

1580 [190] V. Santos-Rosales, A. Iglesias-Mejuto, C.A. García-González, Solvent-Free Approaches 1581 for the Processing of Scaffolds in Regenerative Medicine, Polymers 12(3) (2020) 533.

1582 [191] B. Sultankulov, D. Berillo, K. Sultankulova, T. Tokay, A. Saparov, Progress in the 1583 development of chitosan-based biomaterials for tissue engineering and regenerative medicine, 1584 Biomolecules 9(9) (2019) 470. 
1585 [192] S. Midha, M. Dalela, D. Sybil, P. Patra, S. Mohanty, Advances in three-dimensional 1586 bioprinting of bone: Progress and challenges, Journal of tissue engineering and regenerative 1587 medicine 13(6) (2019) 925-945. 